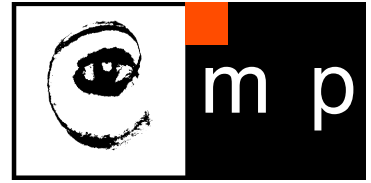

CENTER FOR

MACHINE PERCEPTION

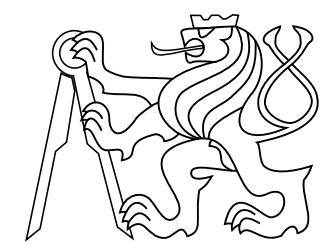

CZECH TECHNICAL UNIVERSITY IN PRAGUE

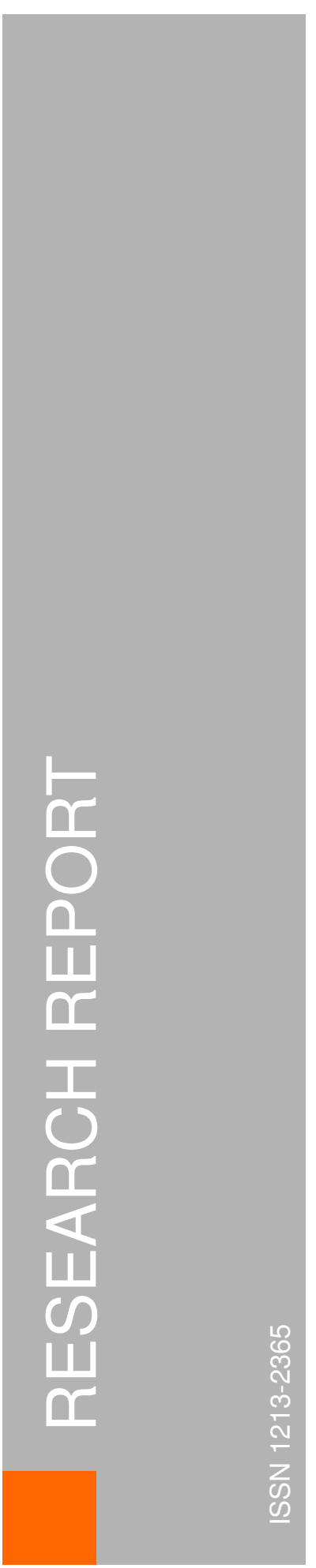

\section{Two-view Matching with View Synthesis Revisited}

\author{
Dmytro Mishkin, Michal Perdoch, Jiri Matas \\ ducha.aiki \{at\}gmail.com,perdom1,matas $\{$ at\} (cmp.felk.cvut.cz) \\ CTU-CMP-2013-15
}

October 29, 2018

The authors were supported by The Czech Science Foundation Project GACR P103/12/G084 and by the Technology Agency of the Czech Republic research program TE01020415 (V3C - Visual Computing Competence Center).

Research Reports of CMP, Czech Technical University in Prague, No. 15, 2013 Published by

Center for Machine Perception, Department of Cybernetics Faculty of Electrical Engineering, Czech Technical University Technická 2, 16627 Prague 6, Czech Republic fax +42022435 7385, phone +42022435 7637, www: http://cmp.felk.cvut.cz 



\title{
Two-view Matching with View Synthesis Revisited
}

\author{
Dmytro Mishkin, Michal Perdoch, Jiri Matas
}

October 29, 2018

\begin{abstract}
Wide-baseline matching focussing on problems with extreme viewpoint change is considered. We introduce the use of view synthesis with affine-covariant detectors to solve such problems and show that matching with the Hessian-Affine or MSER detectors outperforms the state-of-the-art ASIFT [18].

To minimise the loss of speed caused by view synthesis, we propose the Matching On Demand with view Synthesis algorithm (MODS) that uses progressively more synthesized images and more (timeconsuming) detectors until reliable estimation of geometry is possible. We show experimentally that the MODS algorithm solves problems beyond the state-of-the-art and yet is comparable in speed to standard wide-baseline matchers on simpler problems.

Minor contributions include an improved method for tentative correspondence selection, applicable both with and without view synthesis and a view synthesis setup greatly improving MSER robustness to blur and scale change that increase its running time by $10 \%$ only.
\end{abstract}

\section{Introduction}

The standard method for wide baseline matching involves detection of local features, calculation of descriptors, generation of tentative correspondences and their geometric verification using the homography or epipolar constraint.

It is well known [17, 8, 7] that performance of the pipeline decreases in the presence of viewpoint and scale changes, blur, compression artefacts, etc. Lepetit and Fua [12] showed that matching robustness is improved by synthesis of additional views given a single, fronto-parallel view of an object. Morel and $\mathrm{Yu}$ [18] combined viewpoint synthesis with the similarity-covariant Difference-of-Gaussians detector (DoG) and SIFT matching [14]. The resulting image matching method, called ASIFT, successfully matched challenging image pairs with significantly different viewing angles.

We develop the idea of view synthesis for wide baseline matching and propose a number of novelties that improve several stages of the matching pipeline. Some of the improvements are also applicable to two-view matching without synthesis. The proposed MODS wide-baseline matcher ${ }^{1}$ outperforms ASIFT in terms of speed, the number and percentage of correct matches generated as well as in the precision of the estimated geometry. Performance was tested mainly on image pairs with extreme viewpoint changes, but viewpoint synthesis also improves matching results in the presence of phenomena like blur, occlusion and scale change. The following contributions are made: first, we show that the seemingly counter-intuitive synthesis of affine views for "affine-covariant" detectors greatly improves their performance in wide baseline matching. With suitable detector-specific configurations of synthesized viewpoints, found through extensive experimentation, both the Hessian-Affine [16] and MSER [15] detectors clearly outperform DoG [14].

Second, we generalize the "first-to-second-closest SIFT distance ratio" criterion for the selection of tentative correspondences. Depending on the image, the new criterion gives 5-20\% more true matches than the standard at no extra computation cost. The proposed criterion improves even matching performance without synthesis, especially in images with local symmetries.

Third, we propose an adaptive algorithm for matching very challenging image pairs which follows the "do only as much as needed" principle. The MODS algorithm (Matching On Demand with view Synthesis)

\footnotetext{
${ }^{1}$ Available at http://cmp.felk.cvut.cz/wbs/index.html
} 


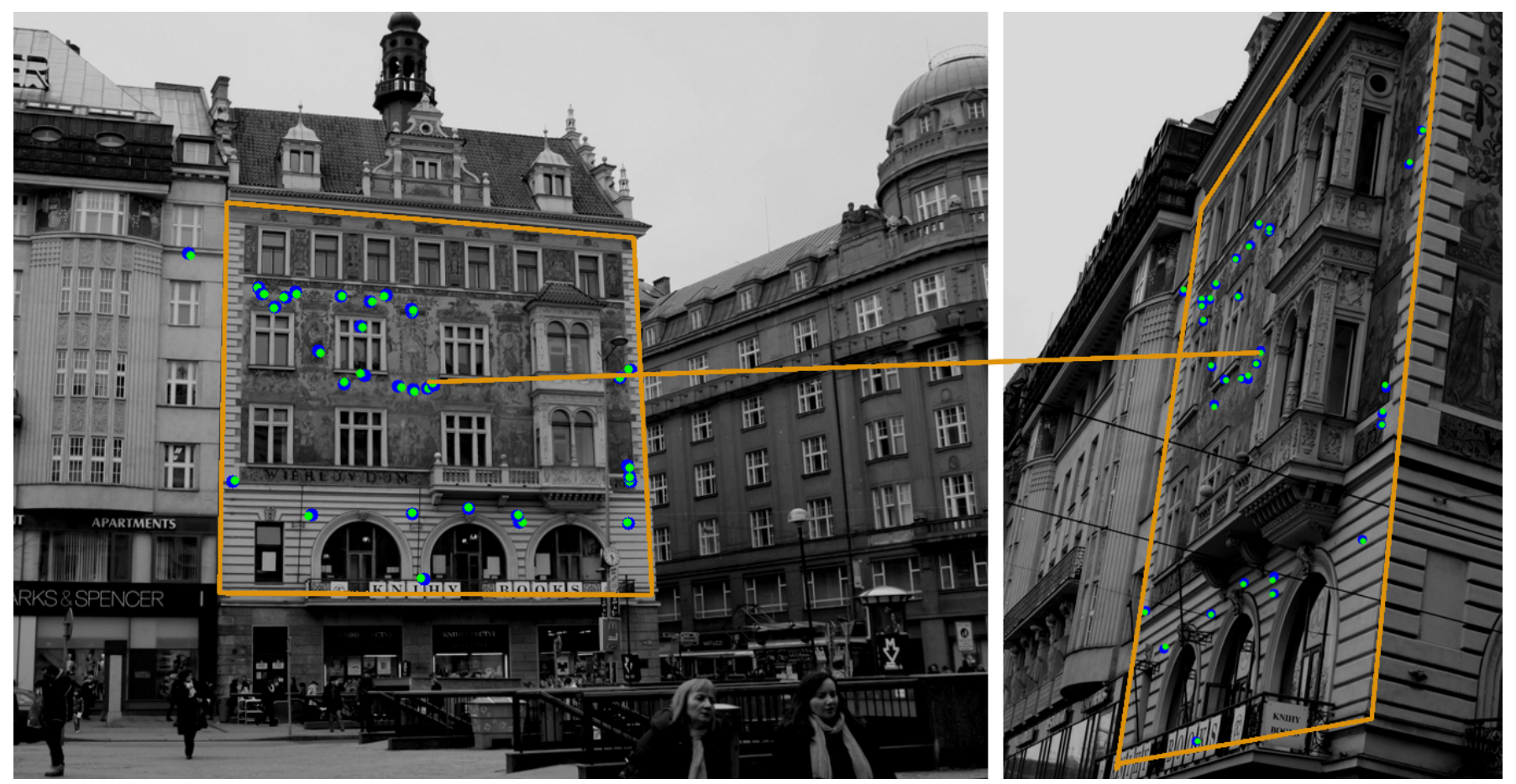

Figure 1. Homography estimation with extreme viewpoint change. The proposed MODS algorithm produces 32 matches, 25 are correct. The state-of-the-art ASIFT [18] outputs 41 matches, 3 are correct. Blue dots: centers of detected regions. Green dots: reprojected centers of corresponding regions showing good alignment.

uses progressively more detector types and more synthesized images until enough correspondences for reliable estimation of two-view geometry are found. MODS is fast on easy image pairs without compromising performance on the hardest problems.

\subsection{Related work}

The use of view synthesis for image matching is a recent development and the literature is limited and includes mainly modifications of the ASIFT algorithm. Liu et al. [13] synthesised perspective warps rather than affine. Pang et al. [20] replaced SIFT by SURF [3] in the ASIFT algorithm to reduce the computation time. Sadek et al. [22] present a new affine covariant descriptor based on SIFT which can be used with or without view synthesis. Detection of the MSERs on the scale space pyramid was proposed by Forssén and Lowe [9].

The rest of the paper is organised in a top-down manner. In Section 2, we introduce the adaptive MODS two-view matching algorithm. Section 3 studies view synthesis for affine-covariant detectors. Experiments are presented in Section 4. Full experimental data is in Appendix.

\section{Matching with On Demand View Synthesis}

The iterative MODS algorithm (see Alg. 1) repeats a sequences of two-view matching procedures, until a required minimum number of geometrically verified correspondences is found. In each iteration, a different detector is used and a different set of views generated. The adopted sequence is an outcome of extensive experimentation with the objective of solving the most challenging problems while keeping speed comparable to standard single-detector wide-baseline matchers for simple problems. For instance, the first iteration of the MODS algorithm runs the MSER detector with only a very coarse scale space pyramid which is $10 \%$ slower than standard MSER. Subsequent iterations run complementary detectors with a higher number of synthesized views. Details on the chose configuration and the selection process are given in Section 3 . The rest of the section describes the steps employed in the iterations of the MODS algorithm. 


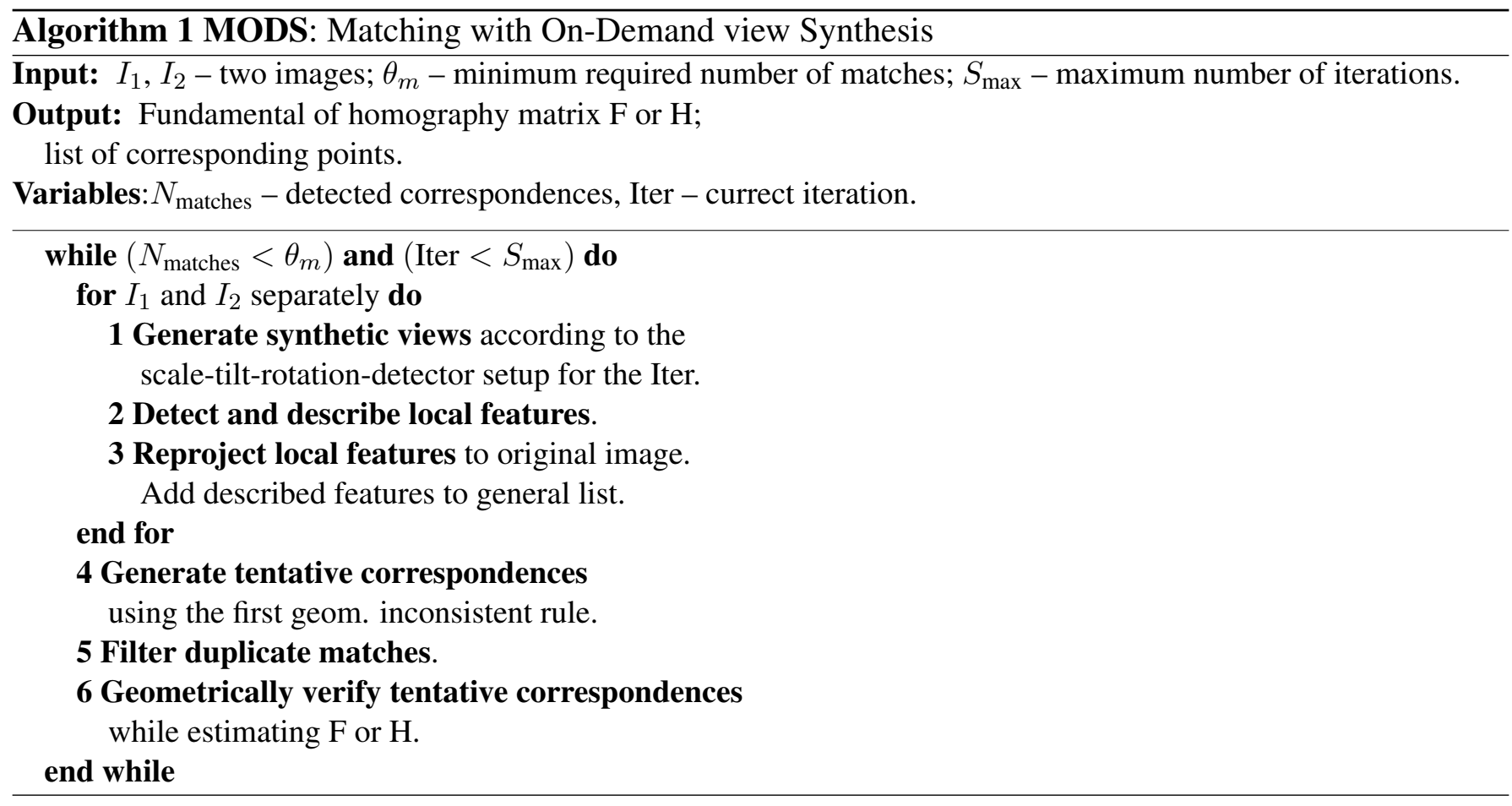

\subsection{Synthetic views generation}

It is well known that a homography $H$ can be approximated by an affine transformation $A$ at a point using the first order Taylor expansion. Further, an affine transformation can be uniquely decomposed by SVD into a rotation, skew, scale and rotation around the optical axis [10]. Morel and Yu [18] proposed to decompose the affine transformation $A$ as

$$
\begin{aligned}
A & =H_{\lambda} R_{1}(\psi) T_{t} R_{2}(\phi)= \\
& =\lambda\left(\begin{array}{cc}
\cos \psi & -\sin \psi \\
\sin \psi & \cos \psi
\end{array}\right)\left(\begin{array}{ll}
t & 0 \\
0 & 1
\end{array}\right)\left(\begin{array}{cc}
\cos \phi & -\sin \phi \\
\sin \phi & \cos \phi
\end{array}\right)
\end{aligned}
$$

where $\lambda>0, R_{1}$ and $R_{2}$ are rotations, and $T_{t}$ is a diagonal matrix with $t>1$. Parameter $t$ is called the absolute tilt, $\phi \in\langle 0, \pi)$ is the optical axis longitude and $\psi \in\langle 0,2 \pi)$ is the rotation of the camera around the optical axis. Each synthesised view is parametrised by the tilt, longitude and optionally the scale and represents a sample of the view-sphere resp. view-volume around the original image.

The view synthesis proceeds in the following steps: at first, scale synthesis is performed by building a Gaussian scale-space with Gaussian $\sigma=\sigma_{\text {base }} \cdot S$ and downsampling factor $S(S<1)$. Second, each image in the scale-space is in-plane rotated by longitude $\phi$ with step $\Delta \phi=\Delta \phi_{\text {base }} / t$. In the third step, all rotated images are convolved with a Gaussian filter with $\sigma=\sigma_{\text {base }}$ along vertical direction and $\sigma=t \cdot \sigma_{\text {base }}$ along horizontal direction to eliminate aliasing in the final tilting step. The tilt is applied by shrinking the image along the horizontal direction by factor $t$. The parameters of the synthesis are: the set of scales $\{S\}, \Delta \phi_{\text {base }}$ - the step of longitude samples at tilt $t=1$, and a set of simulated tilts $\{\mathrm{t}\}$.

\subsection{Local feature detection and description}

The goal of the view synthesis procedure is to provide detectors with a sufficiently similar subset of all artificial views on the view-sphere that allows matching. For affine-covariant detectors, unlike the similaritycovariant DoG of ASIFT, the number of necessary view samples is significantly decreased while the performance for the most difficult image pairs gets improved. Moreover, it is known that different detectors are suitable for different types of images [17] and that some detectors are complementary in the feature points they detect [1]. Our experiments show (c.f. Section 4) that combining detectors improves the overall robustness and speed of the matching procedure.

MODS uses the state-of-the-art affine covariant detectors MSER and Hessian-Affine. The normalised patches are described by the recent modification of SIFT [14] - the RootSIFT [2]. The local feature frames 

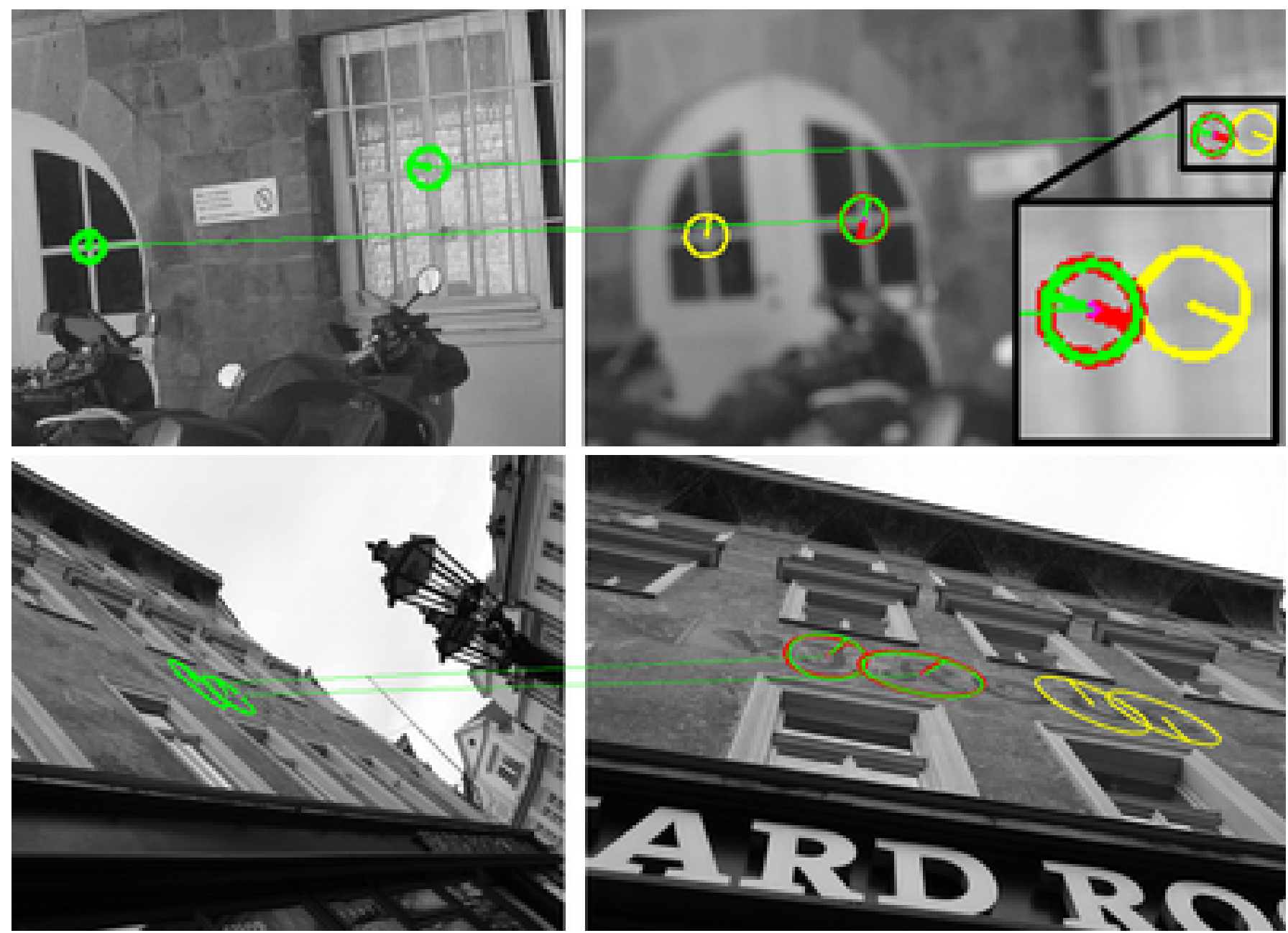

Figure 2. Comparison of the proposed first to 1st inc. ratio matching strategy and the standard first to second closest ratio matching strategy. Red regions are the second closest descriptors, yellow regions correspond to the closest geometrically inconsistent descriptors, green are the true corresponding regions. Upper pair - rotationally symmetric DoG regions, lower pair - affine covariant MSER regions.

computed on the synthesised views are backprojected to the coordinate system of the original image by a known affine matrix $A$ and associated with the descriptor and the originating synthetic view.

\subsection{Tentative correspondence generation}

Different strategies for computation of the tentative correspondences in wide-baseline matching have been proposed. The standard method for matching SIFT(-like) descriptors is based on the distance ratio of the closest to the second closest descriptors in the other image [14]. Performance of this test in general very efficient method degrades when multiple observations of the same feature are present. In this case, the similar descriptors will lead to the first to second SIFT ratio to be close to 1 and the correspondences will "annihilate" each other, despite the fact they all represent the same geometric constraints and are therefore not mutually contradictory (see Figure 2). The problem of multiple detections is amplified in the matching by view synthesis since covariantly detected local features have often a response in multiple synthetic views. We propose to use, instead of comparing the first to the second closest descriptor distance, the distance of the first descriptor and the closest descriptor that is geometrically inconsistent with the first one (denoted 1st inc. in the following). We call descriptors in one image geometrically inconsistent if the Euclidean distance between centers of the regions is $\geq 10$ pixels. The difference of the first-to-second closest ratio strategy and the closest-to-1st inc. strategy is illustrated in Figure 2.

The kd-tree algorithm from FLANN library [19] effectively finds the N-closest descriptors in the other image. The distance ratio thresholds of the closest to 1 st inc. were experimentally selected based on the CDFs of matching and non-matching descriptors (see Appendix A). We recommend to use the same values for SIFT and RootSIFT descriptors, but different thresholds for the different local feature detectors: $R_{\mathrm{MSER}}=0.85, R_{\mathrm{DoG}}=0.85$ and $R_{\mathrm{HA}}=0.8$. 


\subsection{Duplicate filtering}

The redetection of covariant features in synthetic views results in duplicates in tentative correspondences. The duplicate filtering is an optional step and prunes correspondences with close spatial distance of local features in both images. The number of pruned correspondences can be however used later for evaluating the quality (probability of being correct) in PROSAC-like [4] geometric verification.

\subsection{Geometric verification}

The LO-RANSAC [11] algorithm searches for the maximal set of geometrically consistent tentative correspondences. The model of the transformation is set either to homography or epipolar geometry, or automatically determined by a DegenSAC [5] procedure.

\section{View synthesis for affine covariant detectors}
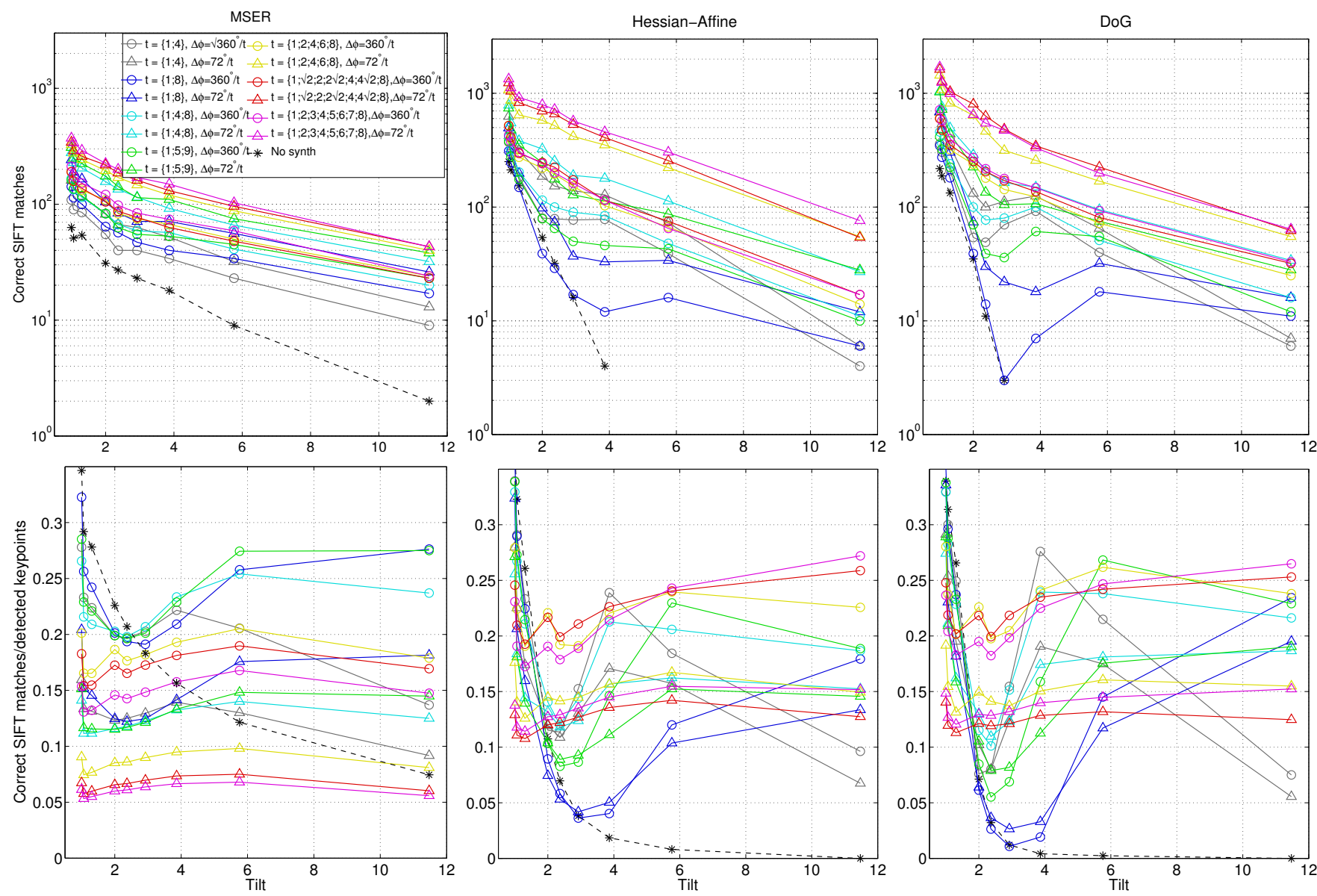

Figure 3. Comparison of view synthesis configurations on the synthetic dataset. First row: the number of correct SIFT matches a robust minimum (value $4 \%$ quantile) over 100 random images from [21]). Second row: the ratio of the number of correct matches to the number of detected regions; the mean over 100 random images. Only selected configurations are shown, full version in Appendix.

Configurations. The first two parameters of the view synthesis, tilt $\{\mathrm{t}\}$ sampling and latitude step $\Delta \phi_{\text {base }}$, were explored in the following synthetic experiment. For each of 100 random images from Oxford Building Dataset] [21], a set of simulated views with latitudes angles $\theta=(0,20,40,60,65,70,75,80,85)^{\circ}$, corresponding to tilt series $t=(1.00,1.06,1.30,2.00,2.36,2.92,3.86,5.75,11.47)^{3}$ was generated. The ground truth affine matrix $A$ was computed for each synthetic view using equation (1) and used in the final

\footnotetext{
${ }^{2}$ available at http://www.robots.ox.ac.uk/ vgg/data/oxbuildings/

${ }^{3}$ assuming that the original image is in the fronto-parallel view
} 

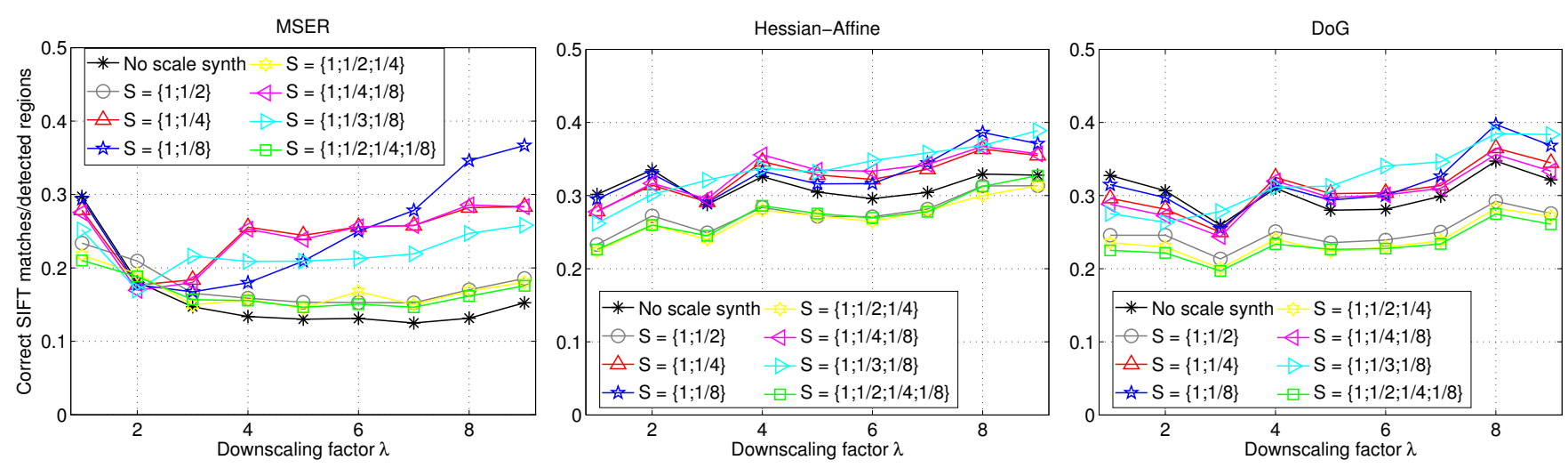

Figure 4. Estimation of the suitable scale synthesis configurations on the synthetic dataset. Ratio of the number of correct matches to the number of detected regions, mean over 100 random images from [21].

Table 1. View synthesis configurations based on the analysis of the algorithm on the synthetic dataset

\begin{tabular}{|c|c|c|}
\hline & \multicolumn{2}{|c|}{ Configurations } \\
\hline Detector & SPARSE & DENSE \\
\hline MSER & $\begin{array}{l}\{S\}=\{1 ; 0.25 ; 0.125\},\{t\}=\{1 ; 5 ; 9\} \\
\Delta \phi=360^{\circ} / t\end{array}$ & $\begin{array}{l}\{S\}=\{1 ; 0.25 ; 0.125\},\{t\}=\{1 ; 2 ; 4 ; 6 ; 8\} \\
\Delta \phi=72^{\circ} / t\end{array}$ \\
\hline HessAff & $\begin{array}{l}\{S\}=\{1\},\{t\}=\{1 ; \sqrt{2} ; 2 ; 2 \sqrt{2} ; 4 ; 4 \sqrt{2} ; 8\} \\
\Delta \phi=360^{\circ} / t\end{array}$ & $\{S\}=\{1\},\{t\}=\{1 ; 2 ; 4 ; 6 ; 8\}, \Delta \phi=72^{\circ} / t$ \\
\hline DoG & $\{S\}=\{1\},\{t\}=\{1 ; 2 ; 4 ; 6 ; 8\}, \Delta \phi=120^{\circ} / t$ & $\begin{array}{l}\{S\}=\{1\},\{t\}=\{1 ; \sqrt{2} ; 2 ; 2 \sqrt{2} ; 4 ; 4 \sqrt{2} ; 8\}, \\
\Delta \phi=72^{\circ} / t\end{array}$ \\
\hline
\end{tabular}

verification step of the MODS algorithm. The various configurations of the view synthesis were tested and results for the selected configurations are shown in Figure 3. Note that the view synthesis significantly increases the matching performance, however after reaching some density of the view-sphere sampling additional views does not bring more correspondences. MSER and Hessian-Affine need sparser viewsphere sampling than DoG.

A similar experiment was performed to find the scale sampling set $\{S\}$ of the view synthesis. Instead of tilting and rotating the images, a synthetic downsampling of the image by a factor $\lambda=1$ to 9 was employed (see Figure 4). It shows that MSER detector is prone to scale changes while the Hessian-Affine and DoG detectors perform well even without view synthesis with scale sampling. Consequently, the benefit of the scale sampling is higher for MSER than for Hessian-Affine and DoG detectors. Tilting and rotation parameters were not used in this experiment i.e. fixed to $\{\mathrm{t}\}=\{1\}$ and $\Delta \phi_{\text {base }}=180$.

Two configurations, SPARSE and DENSE, were chosen for each detector (see Table 1) using the following criteria: efficiency - the ratio of correct matches per detected region, matching performance - the number of unique (non-duplicated) matches on the synthetic image pairs with $85^{\circ}$ out of plane rotation. The SPARSE configuration is fast but still able to solve synthetic image pairs with up to $85^{\circ}$ out of plane rotation. The DENSE configuration generates sufficient number of correspondences for the most image pairs in the EVD dataset for each detector.

Image pre-smoothing. Parameter $\sigma_{\text {base }}$, the amount of image smoothing prior to view synthesis was set experimentally; it affects matching performance significantly. Values too small fail to prevent aliasing, values too high oversmooth the image reducing the number of detected regions. Unlike MSER, the scale-space based detectors like DoG, Hessian-Affine apply pre-smoothing as an initial step. This leads to different optimal values for different detectors. We set $\sigma_{\text {base }}=0.8,0.2$, and 0.4 for the MSER, Hessian-Affine and DoG detectors, respectively. 

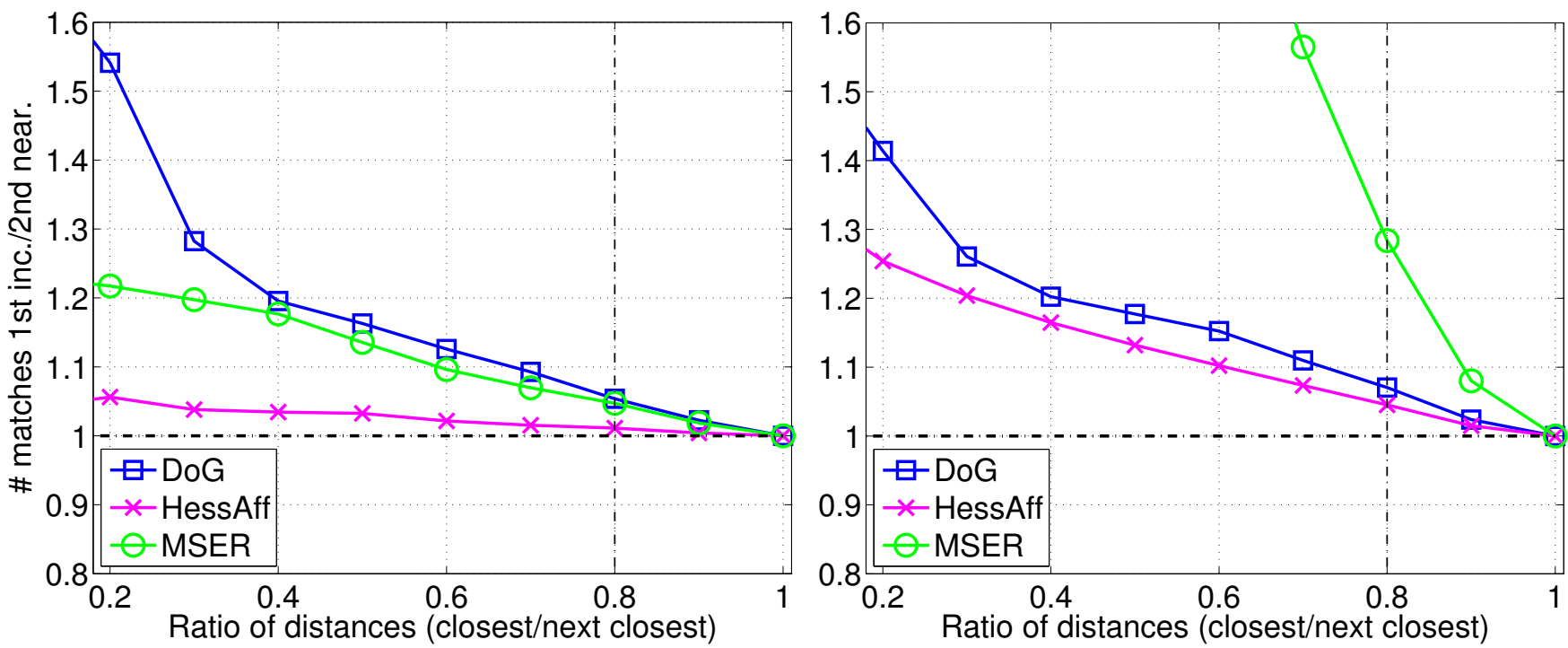

Figure 5. The ratio of the number of correct matches obtained by the 1st inconsistent and 2nd nearest method, without (left) and with (right) view synthesis. The black dashed line denotes the widely used distance ratio threshold $=0.8$.

\section{Experiments}

\subsection{1st geometrically inconsistent vs. 2nd nearest neighbour correspondence selec- tion strategy}

The first to first geometrically inconsistent strategy was evaluated on 50 image pairs of the publicly available datasets [17] and [6]. The cumulative distributions of the number of correct tentative correspondences as functions of the descriptor distance ratio are used for comparison. The new matching strategy improves the performance by up to $5 \%$ for the matching without view synthesis and up to $30 \%$ (see Figure 5 ) for matching with view synthesis at almost no additional computational costs.

\subsection{Results on the Extreme Viewpoint Dataset}

We introduce a two-view matching evaluation datase $\left.\right|^{4}$ with extreme viewpoint changes, see Table 2 . The dataset includes image pairs from publicly available datasets: ADAM and MAG [18], GRAF [17] and THERE [6]. The ground truth homography matrices were estimated by LO-RANSAC using correspondences from all three detectors in view synthesis configuration $\{t\}=\{1 ; \sqrt{2} ; 2 ; 2 \sqrt{2} ; 4 ; 4 \sqrt{2} ; 8\}, \Delta \phi=72^{\circ} / t$. The number of inliers for each image pair was $\geq 50$ and the homographies were manually inspected. For the image pairs GRAF and THERE precise homographies are provided by Cordes et al. [6]. Transition tilts $\tau$ were computed using equation (1) with SVD decomposition of the linearised homography at center of the first image of the pair (see Table 2).

The configurations evaluated are specified in Table 1 . For comparison, ASIFT 5 results are added. Computations were performed on Intel i5 CPU @ 2.6GHz with 4Gb RAM; results for computation on one core are provided. Based on results of the different configuration, we have chosen the following configuration for MODS w.r.t increasing computation time and performance of the configurations - see Table 3. Please note that only views complementary to the previous iterations are synthesised.

The MODS algorithm allows to set the minimum desired number of inliers as a stopping criterion. The recommended value - 15 inliers to the homography, have a very low probability to be a random result, but are few enough to show the time gain from the algorithm. To maximize the number of inliers for each of the detectors, we recommend to use DENSE configuration as a single step. Figure 6 and Table 4 compare the different view synthesis configurations and the "affine-covariant" detectors - they generate more correct matches in a shorter time than the DoG detector. The DoG based matching and ASIFT matching cannot

\footnotetext{
${ }^{4}$ Available at http://cmp.felk.cvut.cz/wbs/index.html

${ }^{5}$ Reference code from http://demo.ipol.im/demo/my_affine_sift
} 
Table 2. The Extreme View Dataset - EVD. Image sources: C - Cordes et al. [6], Ox - Mikolajczyk et al. [17], M Morel and $\mathrm{Yu}$ [18].

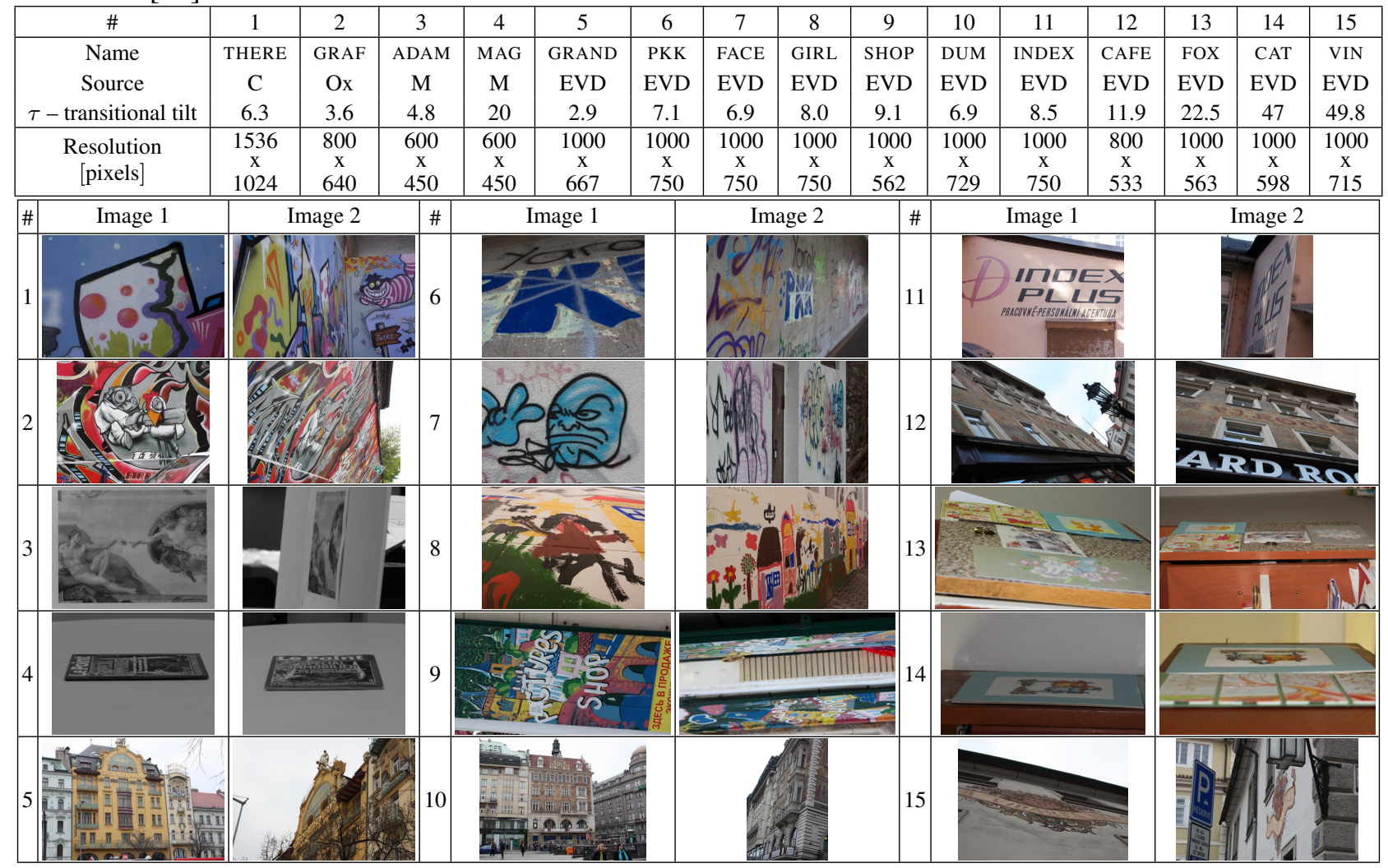

Table 3. Configurations for MODS steps

\begin{tabular}{|c|c|}
\hline Iter. & \multicolumn{1}{c|}{ Setup } \\
\hline 1 & MSER, $\{S\}=\{1 ; 0.25 ; 0.125\},\{t\}=\{1\}, \Delta \phi=360^{\circ} / t$ \\
\hline 2 & MSER, $\{S\}=\{1 ; 0.25 ; 0.125\},\{t\}=\{1 ; 5 ; 9\}, \Delta \phi=360^{\circ} / t$ \\
\hline 3 & HessAff, $\{S\}=\{1\},\{t\}=\{1 ; \sqrt{2} ; 2 ; 2 \sqrt{2} ; 4 ; 4 \sqrt{2} ; 8\}, \Delta \phi=360^{\circ} / t$ \\
\hline 4 & HessAff, $\{S\}=\{1\},\{t\}=\{1 ; 2 ; 4 ; 6 ; 8\}, \Delta \phi=72^{\circ} / t$ \\
\hline
\end{tabular}

solve 3 resp. 9 out of the 15 image pairs. The ASIFT algorithm generates a lower number of correct inliers and works slower than our DoG DENSE configuration (which has the same tilt-rotation set). The main causes are elimination of "one-to-many", including correct, correspondences, the inferiority of the standard 2nd closest ratio and a simple bruteforce algorithm of matching used in ASIFT.

No single detector solved all image pairs. The Hessian-Affine with DENSE configuration successfully solved 14 out of 15 image pairs and outperformed other detectors and configurations in the number of inliers, however, at the expense of the highest computational cost. MSER with no synthesis and in the SPARSE configuration is the fastest and could solve 10 out of 15 image pairs. The MODS algorithm solves all image pairs and saves computational time on processing of the easy pairs at the cost of a small matching overhead on the hard cases. Also, MODS is the fastest algorithm in 7 cases, and in another 2 cases it is just $\sim 10 \%$ slower than the fastest configuration. The difference results of MODS step 2 and MSER SPARSE are caused by randomization in RANSAC and kd-tree building.

Fig. 7] shows the breakdown of the computational time. SIFT description with the dominant orientation estimation take $50 \%$ of the time. Note that the whole process is almost linear in the area of the synthesised views. The only super-linear part, matching, takes only $10 \%$ of the time.

\subsection{MSER vs. blur and scale change}

We have tested performance of recommended scale synthesis configuration for MSER on the image pairs most distorted by blur and scale change from the Oxford [17] dataset. To allow comparison with [17], the standard SIFT was used instead of RootSIFT in this experiment. Note that the results are not fully compatible as we use NN-distance ratio matching threshold $=0.8$ (In [17] no ratio threshold has been 

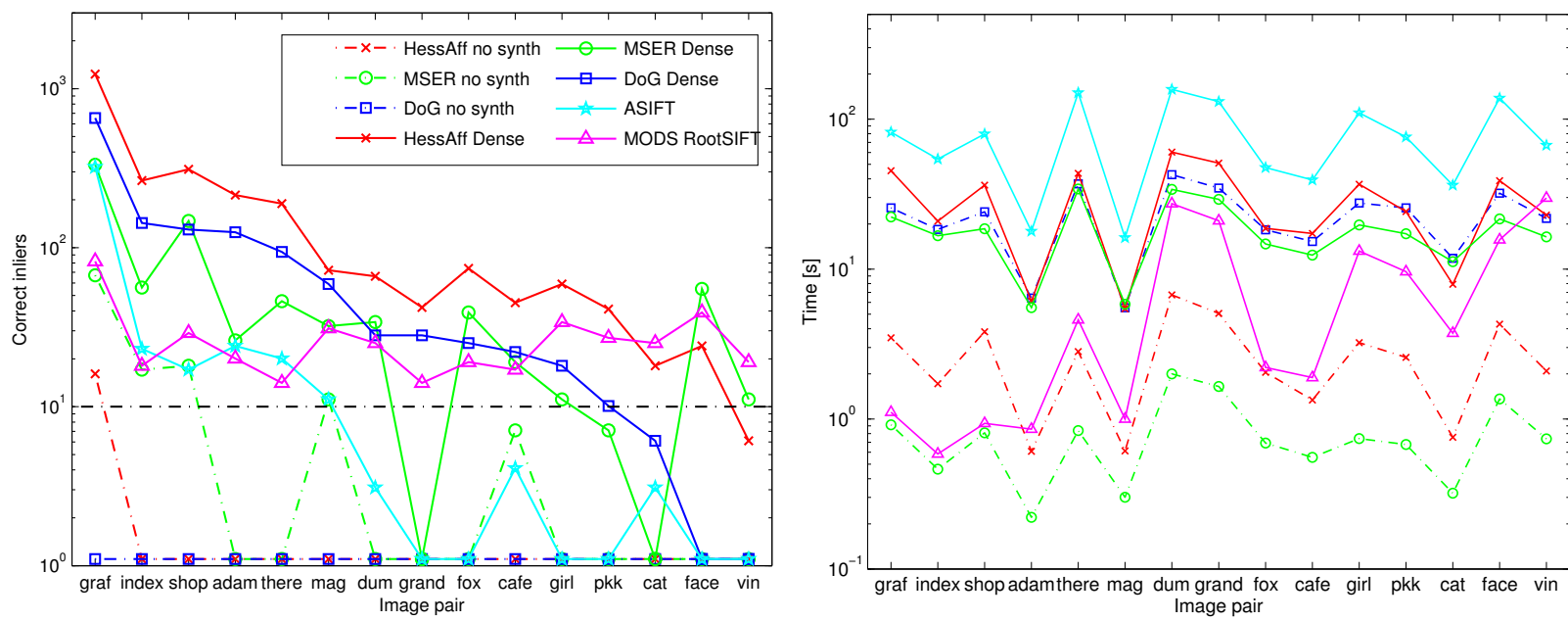

Figure 6. Performance of the selected view synthesis configurations defined in Table 1 . MODS set to find $\geq 15$ inliers. Left - the number of correct RANSAC inliers. The black dashed line marks the level of 10 correct inlier - a minimum for a reliable estimate of two-view geometry. Right - runtime (1 core).

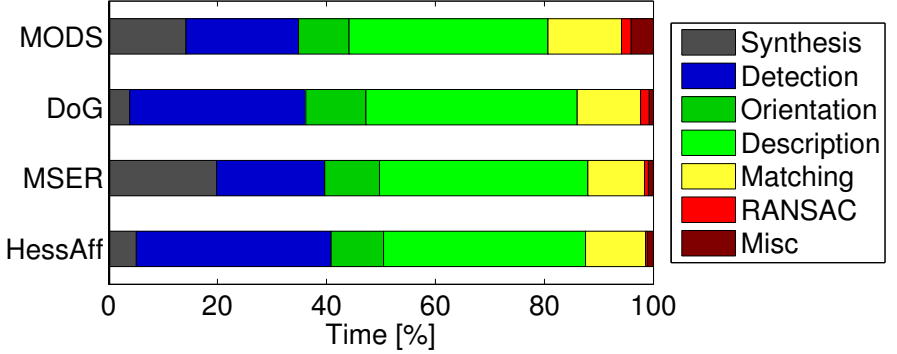

Figure 7. Percentage of time spent in the main stages of the matching with view synthesis process on a single core, DENSE configuration. SIFT description, i.e. the dominant gradient estimation and the descriptor computation is the most time-consuming part.

Table 4. A comparison of different view synthesis and detector configurations (with RootSIFT). Best results are highlighted by a grey background. MODS set to find $\geq 15$ inliers. Results with less than 8 correct inliers are in red.

\begin{tabular}{|c|c|c|c|c|c|c|c|c|c|c|c|c|c|c|c|c|c|c|}
\hline \multirow[t]{2}{*}{ Image } & \multicolumn{6}{|c|}{ Correct inliers } & \multicolumn{6}{|c|}{ Time, 1 core $[s]$} & \multicolumn{6}{|c|}{ Correct inliers/sec } \\
\hline & $\begin{array}{l}n \\
\text { ñ } \\
\text { है } \\
\tilde{n} \\
\tilde{0} \\
\Sigma\end{array}$ & 至 & 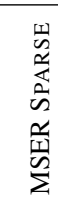 & 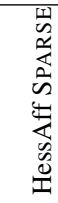 & 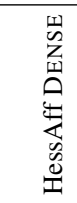 & $\begin{array}{l}\text { 山r } \\
\text { Z } \\
\text { 11 } \\
0 \\
0 \\
0 \\
0\end{array}$ & 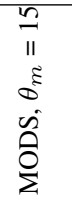 & 点 & 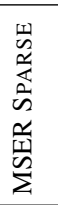 & 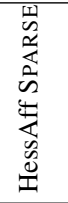 & 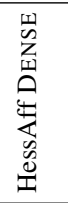 & $\begin{array}{l}\text { In } \\
\text { Z } \\
\text { D̃ } \\
0 \\
0 \\
0\end{array}$ & $\begin{array}{l}n \\
\text { II } \\
\xi \\
\tilde{0} \\
\tilde{n} \\
\hat{0} \\
\tilde{\Sigma}\end{array}$ & $\begin{array}{l}5 \\
\text { 究 }\end{array}$ & 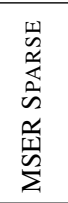 & 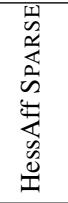 & 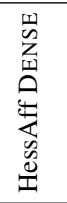 & $\begin{array}{l}\text { II } \\
\tilde{L} \\
Z \\
\text { II } \\
0 \\
0 \\
0\end{array}$ \\
\hline graf & 82 & 322 & 165 & 375 & 1235 & 653 & 1.0 & 81.8 & 3.0 & 11.0 & 45.2 & 25.5 & 83.9 & 3.9 & 55 & 34.1 & 27.3 & 25.6 \\
\hline index & 18 & 23 & 24 & 34 & 264 & 143 & 0.5 & 54.1 & 2.2 & 5.4 & 20.8 & 18.3 & 38.1 & 0.4 & 11.1 & 6.3 & 12.7 & 7.8 \\
\hline shop & 29 & 17 & 73 & 133 & 311 & 130 & 0.8 & 79.5 & 2.5 & 10.1 & 36.2 & 24 & 35.2 & 0.2 & 28.7 & 13.2 & 8.6 & 5.4 \\
\hline adam & 20 & 24 & 18 & 86 & 214 & 125 & 0.8 & 17.8 & 0.7 & 1.6 & 6.0 & 6.3 & 26.7 & 1.3 & 24.3 & 54.1 & 35.6 & 19.8 \\
\hline there & 14 & 20 & 12 & 49 & 189 & 94 & 4.5 & 150.0 & 4.5 & 10.1 & 43.4 & 36.9 & 3.1 & 0.1 & 2.7 & 4.9 & 4.4 & 2.5 \\
\hline mag & 31 & 11 & 28 & 54 & 72 & 59 & 0.8 & 16.1 & 0.8 & 1.6 & 5.3 & 5.4 & 37.3 & 0.7 & 34.4 & 33.5 & 13.5 & 10.9 \\
\hline dum & 25 & 3 & 0 & 10 & 66 & 28 & 29.4 & 158.0 & 4.8 & 20.1 & 60.2 & 42.5 & 0.9 & 0.0 & 0.0 & 0.5 & 1.1 & 0.7 \\
\hline grand & 14 & 0 & 9 & 0 & 42 & 28 & 21.9 & 131.0 & 4.2 & 14.8 & 50.8 & 34.6 & 0.6 & 0.0 & 2.1 & 0.0 & 0.8 & 0.8 \\
\hline fox & 19 & 0 & 19 & 22 & 74 & 25 & 2.1 & 47.4 & 2.1 & 5.8 & 18.6 & 18.2 & 9.0 & 0.0 & 9.3 & 3.8 & 4 & 1.4 \\
\hline cafe & 17 & 4 & 14 & 0 & 45 & 22 & 1.8 & 39.2 & 1.7 & 4.5 & 17.2 & 15.2 & 9.3 & 0.1 & 8.2 & 0.0 & 2.6 & 1.4 \\
\hline girl & 34 & 0 & 0 & 14 & 59 & 18 & 13.1 & 110.0 & 2.7 & 10.0 & 36.7 & 27.5 & 2.6 & 0.0 & 0.0 & 1.4 & 1.6 & 0.7 \\
\hline pkk & 27 & 0 & 6 & 12 & 41 & 10 & 9.5 & 75.9 & 2.4 & 6.8 & 24.1 & 25.5 & 2.8 & 0.0 & 2.5 & 1.8 & 1.7 & 0.4 \\
\hline cat & 25 & 3 & 0 & 21 & 18 & 6 & 3.9 & 36.2 & 1.4 & 2.2 & 7.8 & 11.7 & 6.3 & 0.1 & 0.0 & 9.6 & 2.3 & 0.5 \\
\hline face & 39 & 0 & 9 & 17 & 24 & 0 & 15.6 & 138.0 & 3.4 & 11.3 & 38.8 & 32.0 & 2.5 & 0.0 & 2.7 & 1.5 & 0.6 & 0.0 \\
\hline vin & 19 & 0 & 0 & 0 & 6 & 0 & 30.3 & 66.9 & 2.3 & 6.3 & 22.8 & 21.7 & 0.6 & 0.0 & 0.0 & 0.0 & 0.3 & 0.0 \\
\hline
\end{tabular}

used, so the absolute number of the matches differs a lot. But relative ratio between detectors performance remains the same). We have also performed the duplicate filtering procedure, which reduces the number of correspondences (c.f. Section 2).

Figure 8 shows that scale synthesis with 1 st geom. inconsistent rule improves MSER performance by $60 \%$ to $1000 \%$, solving the most common MSER problems - sensitivity to blur and scale change. The quality of tentative correspondences also increases with the proposed scale synthesis configuration (Figure 8, right). Table 6 shows the computation time. 
Table 5. MODS $\left(\theta_{m}=15\right)$ performance on the EVD dataset. The k-th iteration includes regions from all previous steps.

\begin{tabular}{|c|c|c|c|c|c|c|c|c|c|}
\hline \multirow[t]{3}{*}{ Image } & \multicolumn{9}{|c|}{$\begin{array}{l}\text { MODS (SIFT) } 4 \text { steps. } \\
\text { 1. MSER Scale only. 2. MSER SPARSE. } \\
\text { 3. HessAff SPARSE. 4. HessAff MAX }\end{array}$} \\
\hline & & Time & & RA & $\mathrm{AC}$ & & $\begin{array}{l}\text { entatives } \\
\text { quality }\end{array}$ & Reg & as \\
\hline & 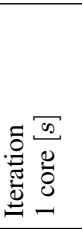 & 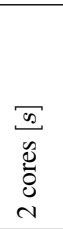 & $\begin{array}{l}\infty \\
\tilde{D} \\
\dot{0} \\
\dot{0} \\
\dot{y}\end{array}$ & 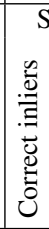 & : & 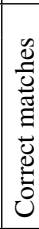 & 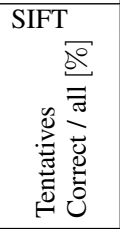 & $\begin{array}{l}\widetilde{\Xi} \\
\mathbb{E} \\
\Xi\end{array}$ & 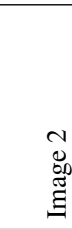 \\
\hline graf & $\begin{array}{ll}1 & 1\end{array}$ & 0.8 & 0.8 & 81 & 85 & 82 & 16051.2 & 1018 & 1674 \\
\hline ex & $\begin{array}{ll}1 & 0.5\end{array}$ & 0.4 & 0.4 & 19 & 20 & 19 & 5633.9 & 4 & 24 \\
\hline shop & $\begin{array}{ll}1 & 0.8\end{array}$ & 30.7 & 0.7 & 28 & 30 & 28 & 8433.3 & 1321 & 711 \\
\hline adam & $\begin{array}{ll}2 & 0.8\end{array}$ & 0.5 & 0.3 & 19 & 22 & 21 & 4843.8 & 357 & 16 \\
\hline there & 24.5 & 2.8 & 2 & 10 & 18 & 15 & 6622.7 & 571 & 2833 \\
\hline mag & $\begin{array}{ll}2 & 0.8\end{array}$ & 30.5 & 0.4 & 30 & 31 & 30 & 5257.7 & 393 & 509 \\
\hline dum & 329.4 & 18.9 & 15.5 & 24 & 29 & & 11362.6 & 33342 & 23711 \\
\hline grand & 321.9 & 13.7 & 11.3 & 17 & 25 & 21 & $\begin{array}{ll}754 & 2.8\end{array}$ & 24731 & 20297 \\
\hline fox & $\begin{array}{ll}2 & 2.1\end{array}$ & 1.4 & 1.1 & 16 & 19 & 19 & 7625 & 1717 & 1011 \\
\hline cafe & $\begin{array}{ll}2 & 1.8\end{array}$ & 31.2 & 0.9 & 18 & 20 & 18 & 14212.7 & 1402 & 1319 \\
\hline girl & $\begin{array}{ll}3 & 13.1\end{array}$ & 7.4 & 5.3 & 35 & 46 & 38 & $549 \quad 6.9$ & 10460 & 16105 \\
\hline pkk & $2 \quad 2.5$ & 51.6 & 1.3 & 7 & 15 & 10 & 8112.3 & 1229 & 1267 \\
\hline cat & $\begin{array}{ll}3 & 3.9\end{array}$ & 2 & 1.4 & 35 & 38 & 35 & 14324.5 & 1262 & 3279 \\
\hline face & 23.6 & 2.4 & 2 & 9 & 15 & 11 & 1189.3 & 3411 & 2371 \\
\hline vin & 430.3 & 17.8 & 12.5 & 18 & 38 & 22 & $657 \quad 3.4$ & 18956 & 31984 \\
\hline
\end{tabular}

\begin{tabular}{|c|c|c|c|c|c|c|c|c|}
\hline \multirow[t]{3}{*}{ Image } & \multicolumn{8}{|c|}{$\begin{array}{l}\text { MODS (RootSIFT), } 4 \text { steps. } \\
\text { ISER Scale only. 2. MSER SPARSE. } \\
\text { HessAff SPARSE. 4. HessAff MAX }\end{array}$} \\
\hline & & Time & & RA & $\mathrm{AC}$ & $\begin{array}{c}\text { Tentatives } \\
\text { quality }\end{array}$ & Regi & ions \\
\hline & 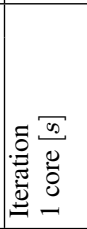 & 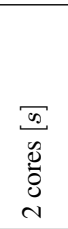 & $\begin{array}{l}\omega \\
\tilde{0} \\
\dot{0} \\
0 \\
\dot{0}\end{array}$ & 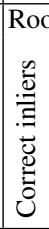 & $\stackrel{\stackrel{\mathscr{\omega}}{\Xi}}{\Xi}$ & 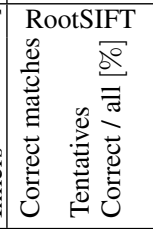 & 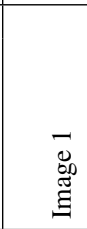 & $\begin{array}{l}N \\
\mathscr{8} \\
\mathbb{Z} \\
\tilde{\Xi}\end{array}$ \\
\hline graf & $\begin{array}{ll}1 & 1\end{array}$ & 0.8 & 0.8 & 82 & 87 & $83 \quad 15453.9$ & 1018 & 1674 \\
\hline index & $\begin{array}{ll}1 & 0.5\end{array}$ & $\begin{array}{ll}5 & 0.4\end{array}$ & 0.4 & 18 & 20 & $18 \quad 4242.9$ & 411 & 246 \\
\hline shop & $\begin{array}{ll}1 & 0.8\end{array}$ & $\begin{array}{ll}8 & 0.7\end{array}$ & 0.7 & 29 & 31 & 296147.5 & 1321 & 711 \\
\hline adam & $\begin{array}{ll}2 & 0.8\end{array}$ & $\begin{array}{ll}8 & 0.5\end{array}$ & 0.4 & 20 & 23 & $22 \quad 4746.8$ & 357 & 164 \\
\hline there & 24.5 & $5 \quad 2.8$ & 2 & 14 & 17 & $16 \quad 6026.7$ & 571 & 2833 \\
\hline mag & $\begin{array}{ll}2 & 0.9\end{array}$ & 90.5 & 0.4 & 31 & 31 & $31 \quad 4470.5$ & 393 & 509 \\
\hline dum & $\begin{array}{ll}3 & 27.2\end{array}$ & 216.9 & 13.5 & 25 & 32 & $29850 \quad 3.4$ & 33342 & 23711 \\
\hline grand & 320.9 & 912.5 & 10 & 14 & 24 & $19468 \quad 4.1$ & 24731 & 20297 \\
\hline fox & $\begin{array}{ll}2 & 2.1\end{array}$ & 11.4 & 1.1 & 19 & 20 & $20 \quad 6232.3$ & 1717 & 1011 \\
\hline cafe & $\begin{array}{ll}2 & 1.8\end{array}$ & $\begin{array}{ll}8 & 1.2\end{array}$ & 0.9 & 17 & 21 & 1811715.4 & 1402 & 1319 \\
\hline girl & 313.1 & 17.3 & 5.2 & 34 & 44 & $38436 \quad 8.7$ & 10460 & 16105 \\
\hline pkk & $\begin{array}{ll}3 & 9.5\end{array}$ & $5 \quad 5.3$ & 4 & 27 & 37 & 333449.6 & 10686 & 7085 \\
\hline cat & $3 \quad 3.6$ & $\begin{array}{ll}6 & 2.1\end{array}$ & 1.5 & 25 & 34 & 3014920.1 & 1262 & 3279 \\
\hline face & 315.6 & $\begin{array}{ll}6 & 8.9\end{array}$ & 7.1 & 39 & 44 & $42534 \quad 7.9$ & 18857 & 13271 \\
\hline vin & 429.7 & 717.1 & 11.8 & 19 & 32 & $21455 \quad 4.6$ & 18956 & 31984 \\
\hline
\end{tabular}
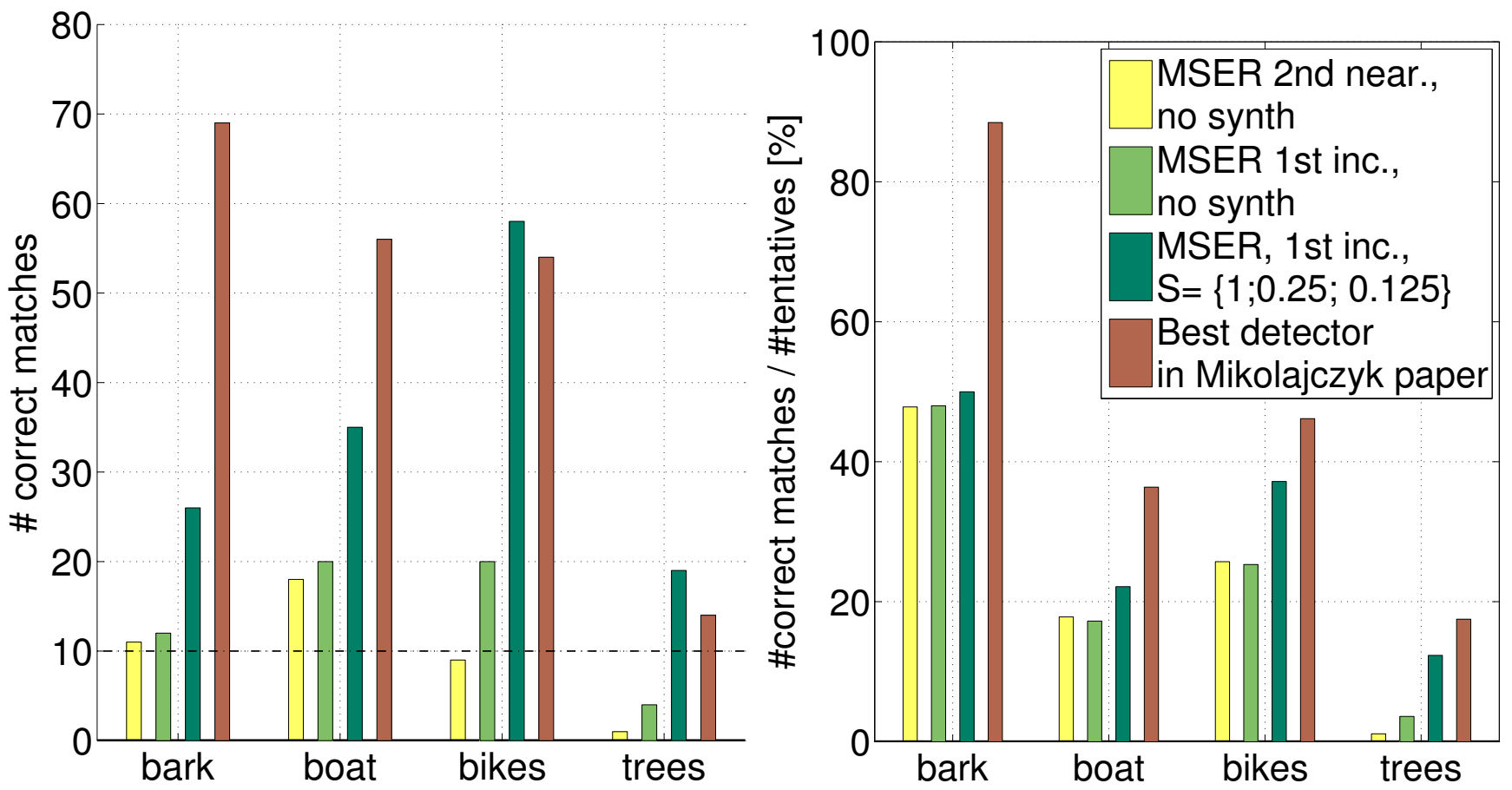

Figure 8. MSER performance with and w/o scale synthesis on the most distorted pairs (1-6) with scale change and blur from [17]. Left - the number of correct SIFT matches. Right - the proportion of correct matches within tentative correspondences. The best detectors from [17]: BARK, BOAT, TREES - Hessian-Affine, BIKES - IBR are shown for comparison.

\section{Conclusions}

We have introduced view synthesis to two-view wide-baseline matching with affine-covariant detectors and shown that matching with the Hessian-Affine or MSER detectors outperforms the state-of-the-art ASIFT.

Table 6. MSER matcher runtime on Oxford [17] dataset

\begin{tabular}{|l|l|}
\hline scale synthesis setup & time $[\mathrm{s}]$ \\
\hline$\{S\}=\{1\}$ & 56.6 \\
$\{S\}=\{1 ; 0.25 ; 0.125\}$ & 61.5 \\
\hline
\end{tabular}


To address the robustness vs. speed trade-off, we have proposed the Matching On Demand with view Synthesis algorithm (MODS) that uses progressively more synthesized images and more (time-consuming) detectors until a reliable estimate of geometry is obtained. We show experimentally that the MODS algorithm solves matching problems beyond the state-of-the-art and yet is comparable in speed to standard wide-baseline matchers on simpler problems.

Minor contributions include an improved method for tentative correspondence selection, applicable both with and without view synthesis. A modification of the standard first to second nearest SIFT distance rule increases the number of correct matches by $5-20 \%$ at no additional computational cost. Finally, we found a simple view synthesis set up costing less than $10 \%$ of time that greatly improves MSER robustness to blur and scale change.

\section{References}

[1] H. Aanaes, A. Dahl, and K. Steenstrup Pedersen. Interesting interest points. IJCV, 97(1):18-35, 2012.

[2] R. Arandjelović and A. Zisserman. Three things everyone should know to improve object retrieval. In CVPR, 2012.

[3] H. Bay, T. Tuytelaars, and L. V. Gool. SURF: Speeded up robust features. In ECCV, 2006.

[4] O. Chum and J. Matas. Matching with PROSAC - progressive sample consensus. In CVPR, 2005.

[5] O. Chum, T. Werner and J. Matas. Two-view geometry estimation unaffected by a dominant plane. In CVPR, 2005.

[6] K. Cordes, B. Rosenhahn, and J. Ostermann. Increasing the accuracy of feature evaluation benchmarks using differential evolution. In SSCI-Symposium on Differential Evolution, 2011.

[7] A. L. Dahl, H. Aanaes, and K. S. Pedersen. Finding the best feature detector-descriptor combination. 3DIMPVT, 2011.

[8] F. Fraundorfer and H. Bischof. A novel performance evaluation method of local detectors on non-planar scenes. In CVPR'05 Workshops, 2005.

[9] P.-E. Forssén and D. Lowe. Shape Descriptors for Maximally Stable Extremal Regions. In ICCV, 2007.

[10] R. I. Hartley and A. Zisserman. Multiple View Geometry in Computer Vision, 2004.

[11] K. Lebeda, J. Matas, and O. Chum. Fixing the Locally Optimized RANSAC. In BMVC, 2012.

[12] V. Lepetit and P. Fua. Keypoint recognition using randomized trees. PAMI, 28(9):1465-1479,2006.

[13] W. Liu, Y. Wang, J. Chen, J. Guo, and Y. Lu. A completely affine invariant image-matching method based on perspective projection. MVA, 23(2):231-242, 2012.

[14] D. Lowe. Distinctive image features from scale-invariant keypoints. IJCV, 60(2):91-110, 2004.

[15] J. Matas, O. Chum, M. Urban, and T. Pajdla. Robust wide-baseline stereo from maximally stable extremal regions. In $B M V C, 2002$

[16] K. Mikolajczyk and C. Schmid. Scale \& affine invariant interest point detectors. IJCV, 60(1):63-86,2004.

[17] K. Mikolajczyk, T. Tuytelaars, C. Schmid, A. Zisserman, J. Matas, F. Schaffalitzky, T. Kadir, and L. V. Gool. A comparison of affine region detectors. IJCV, 65(1-2):43-72, 2005.

[18] J.-M. Morel and G. Yu. ASIFT: A new framework for fully affine invariant image comparison. SIIMS, 2(2):438-469, 2009.

[19] M. Muja and D. G. Lowe. Fast approximate nearest neighbors with automatic algorithm configuration. In VISSAPP'09), 2009.

[20] Y. Pang, W. Li, Y. Yuan, and J. Pan. Fully affine invariant SURF for image matching. Neurocomputing, 85(0):6-10, 2012.

[21] J. Philbin, O. Chum, M. Isard, J. Sivic, and A. Zisserman. Object retrieval with large vocabularies and fast spatial matching. In $C V P R, 2007$.

[22] R. Sadek, C. Constantinopoulos, E. Meinhardt, C. Ballester, and V. Caselles. On affine invariant descriptors related to SIFT. SIIMS, 5(2):652-687,2012. 


\section{Appendix}

\section{A Tuning view synthesis parameters}

Estimating threshold on the distance ratio. The well known [14] matching strategy for SIFT descriptors is based on the distance ratio of the first to the second closest descriptor. The aim of this experiment is to set the threshold of the proposed modification - first to first geometrically inconsistent matching strategy.

To estimate the threshold we used 50 image pairs of the publicly available datasets [17] and [6], all pairs are provided with known homography transformation. The detectors - MSER, Hessian-Affine, DoG - were run on all pairs of images and distances between all descriptors in each pair computed. Then the closest, second closest and closest geometrically inconsistent descriptors were identified. The cumulative distributions of the number of correct and incorrect tentative correspondences as a function of the distance ratio were computed for both strategies using the ground truth homographies.

The results for both SIFT and RootSIFT descriptors are shown in Figure 9. We see that the DoG and MSER features are slightly less discriminative than Hessian-Affine. It is also clear from comparing the left and right columns in Figure 9, that the features detected using view synthesis are less distinctive. However, the distribution of incorrect matches does not change significantly, thus the thresholds for the new strategy with view synthesis can be kept on the value similar to the threshold without view synthesis. The results for the SIFT and RootSIFT descriptors are also very similar. Therefore, we propose to set the threshold of the first to first geometrically inconsistent distance ratio $R$ for the local feature detectors as follows: $R_{\mathrm{MSER}}=0.85, R_{\mathrm{DoG}}=0.85$ and $R_{\mathrm{HA}}=0.8$.

Tilt set and latitude sampling step. The first two parameters of the view synthesis, tilt $\{\mathrm{t}\}$ sampling and latitude step $\Delta \phi_{\text {base }}$, were explored in the following synthetic experiment. For each of 100 random images from Oxford Building Dataset ${ }^{6}[21]$, a set of simulated views with latitudes angles $\theta=(0,20,40$, $60,65,70,75,80,85)^{\circ}$, corresponding to tilt series $t=(1.00,1.06,1.30,2.00,2.36,2.92,3.86,5.75$, 11.47) 7 was generated. The reference image have been convolved with a Gaussian filter with $\sigma_{H}=0.8$ along horizontal direction and $\sigma_{V}=0.8 t$ along vertical direction and finally shrunk in vertical direction by $t$. The ground truth affine matrix $A$ was computed for each synthetic view using equation (1) and used in the final verification step of the MODS algorithm. The various configurations of the view synthesis were tested and results for the selected configurations are shown in Figures $10-12$. Note that the view synthesis significantly increases the matching performance, however after reaching some density of the view-sphere sampling additional views does not bring more correspondences. MSER and Hessian-Affine need sparser view-sphere sampling than DoG.

Two configurations, SPARSE and DENSE, were chosen for each detector (see Table 1) using the following criteria: efficiency - the ratio of correct matches per detected region, matching performance - the number of unique (non-duplicated) matches on the synthetic image pairs with $85^{\circ}$ out of plane rotation. The SPARSE configuration is fast but still able to solve synthetic image pairs with up to $85^{\circ}$ out of plane rotation. The DENSE configuration generates sufficient number of correspondences for the most image pairs in the EVD dataset for each detector.

Image pre-smoothing. The early experiments with view synthesis, have shown that the amount of image smoothing $\sigma_{\text {base }}$ prior to view synthesis affects matching performance significantly. Values too small fail to prevent aliasing, values too high oversmooth the image reducing the number of detected regions. Unlike MSER, the scale-space based detectors - DoG, Hessian-Affine apply pre-smoothing as the initial step of the scale-space pyramid.

This experiment measures the effect of the pre-smoothing parameter $\sigma_{\text {base }}$ on the matching performance of different detectors. The range of values of the $\sigma_{\text {base }}$ were used in matching of 35 image pairs of the publicly available datasets [17] and [6]. We have divided all pairs into two sets "Structured images" - scenes GRAF, GRACE, POSTERS, THERE, UNDERGROUND (25 image pairs in total) from [6] and "Images with repeated textures" - scenes WALL, COLORS (10 image pairs in total) [6], [17]. The DENSE configurations

\footnotetext{
${ }^{6}$ available at http://www.robots.ox.ac.uk/ vgg/data/oxbuildings/

${ }^{7}$ assuming that the original image is in the fronto-parallel view
} 
No view synthesis
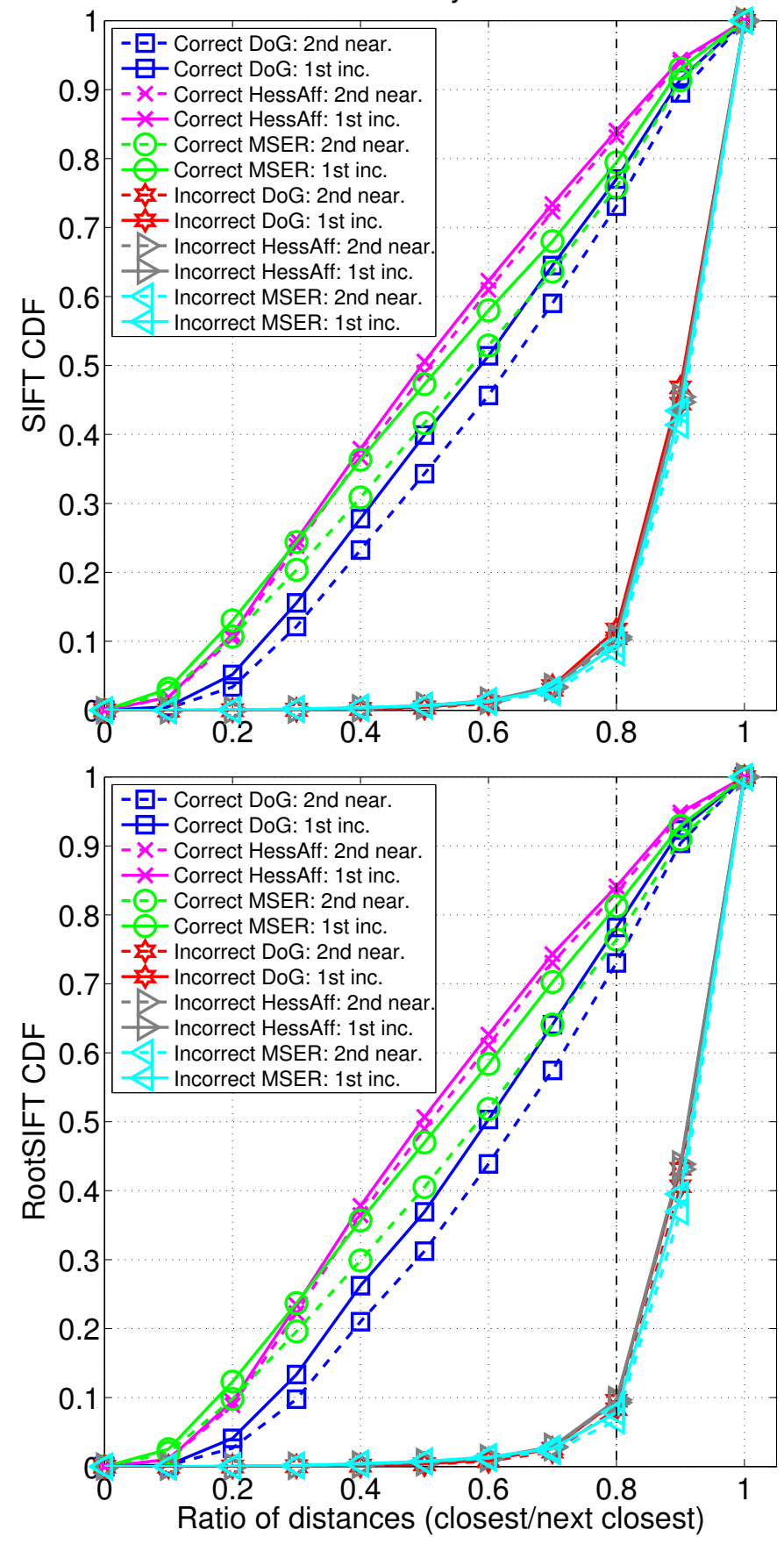

With view synthesis
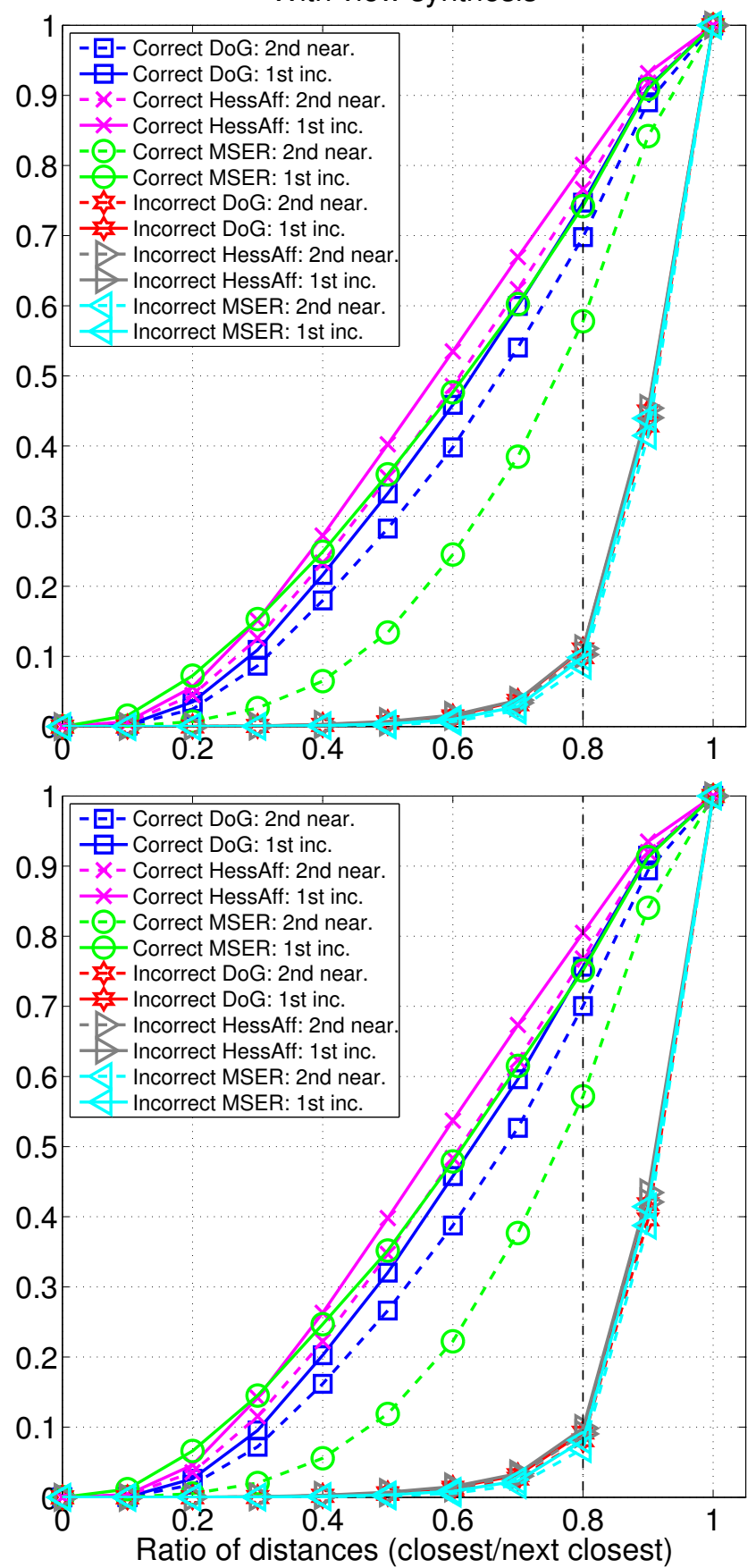

Figure 9. CDF. Columns: left - no view synthesis, right - with view synthesis. Rows: upper - SIFT, lower - RootSIFT. Average over 50 image pairs from Mikolajczyk et al. [17] and Cordes et al. [6] datasets. Black dashed line displays standard threshold $=0.8$.

of the view synthesis were chosen for each of the detectors (see Table 1). Based on this experiment (see Figure 13), we have set following parameters for image pre-smoothing in the MODS algorithm: $\sigma_{\text {base }}=$ 0.8, 0.2, and 0.4 for the MSER, Hessian-Affine and DoG detectors, respectively.

\section{B Full version of the experiments on the EVD dataset}

The full version of experimental evaluation of the matching with view synthesis algorithm on EVD dataset is presented in this section. For this very challenging dataset it is hard to obtain ground truth homographies from the manually selected correspondences. Therefore, the ground truth homography matrices were estimated by running LO-RANSAC on correspondences of all three detectors with the view synthesis con- 
figuration $\{t\}=\{1 ; \sqrt{2} ; 2 ; 2 \sqrt{2} ; 4 ; 4 \sqrt{2} ; 8\}, \Delta \phi=72^{\circ} / t$. The number of inliers for each image pair was $\geq$ 50 and the homographies were manually inspected. For the image pairs GRAF and THERE precise homographies were provided by Cordes et al. [6]. The transition tilts were computed using equation (1) with SVD decomposition of the linearised homography at the center of the first image of the pair. The configurations of detectors evaluated are listed in Table 1, additionally, the performance of the MODS and MSER detector with scale synthesis were compared. The configuration for MODS algorithm is shown in Table 3. The MODS algorithm allows to set the minimum desired number of inliers as a stopping criterion. We set the threshold to 15 inliers, since fifteen inliers to a homography (with duplicate matches removed) have very low probability of being accidental and yet allow to demonstrate the speed gain of the algorithm.

The results for all configurations for all detectors are shown in Tables $7-19$. For comparison, ASIFT ${ }^{8}$ results were added. The timing measurements are reported for single, two and four cores of the Intel i5 CPU @ 2.6GHz processor with 4GB RAM.

\footnotetext{
${ }^{8}$ Reference code from http://demo.ipol.im/demo/my_affine_sift
} 

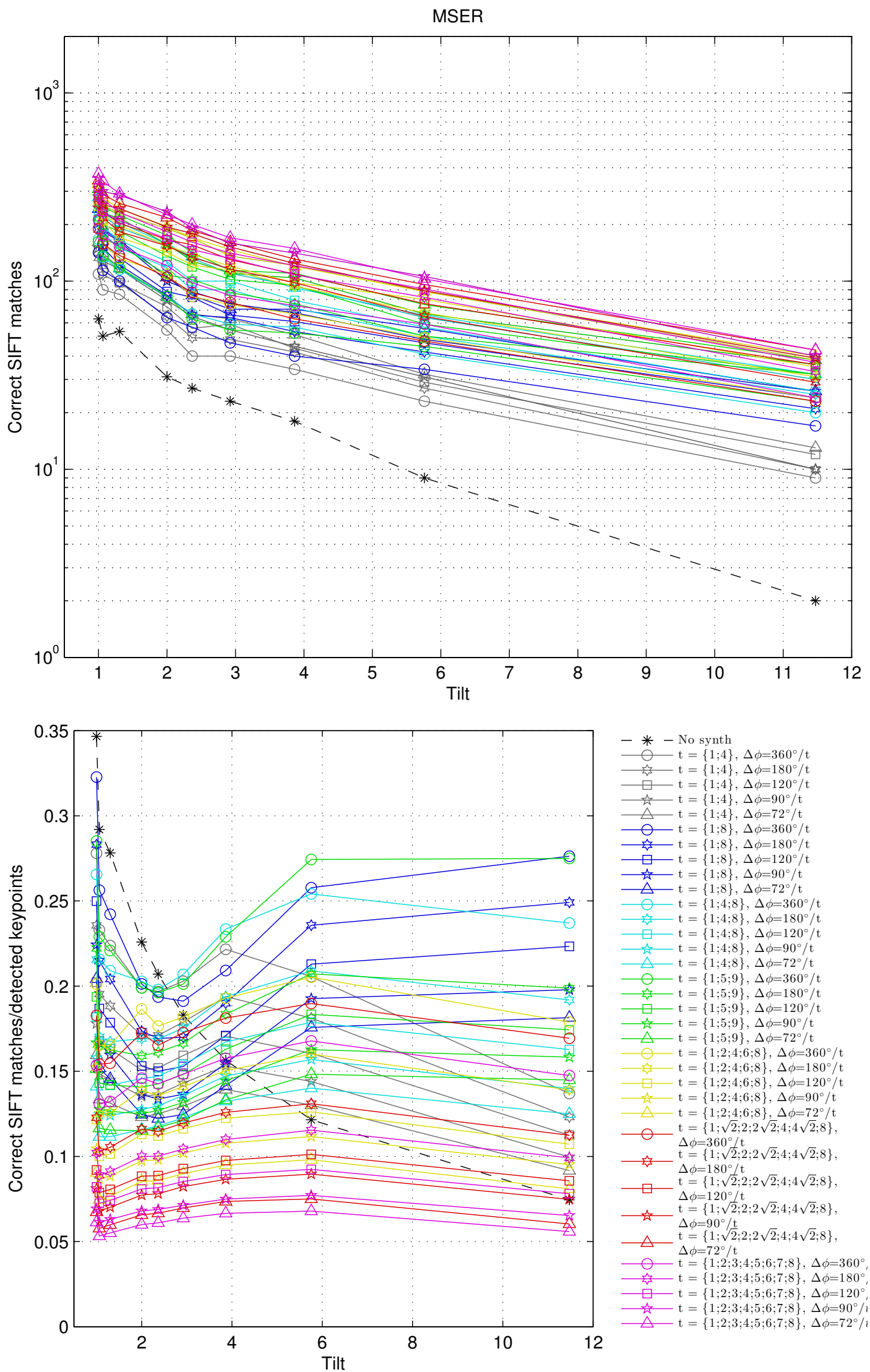

Figure 10. Comparison of MSER view synthesis configurations on the synthetic dataset. Upper graph - the number of correct SIFT matches a robust minimum (value 4\% quantile) over 100 random images from [21]. Lower graph the ratio of the number of correct matches to the number of detected regions; the mean over 100 random images. 

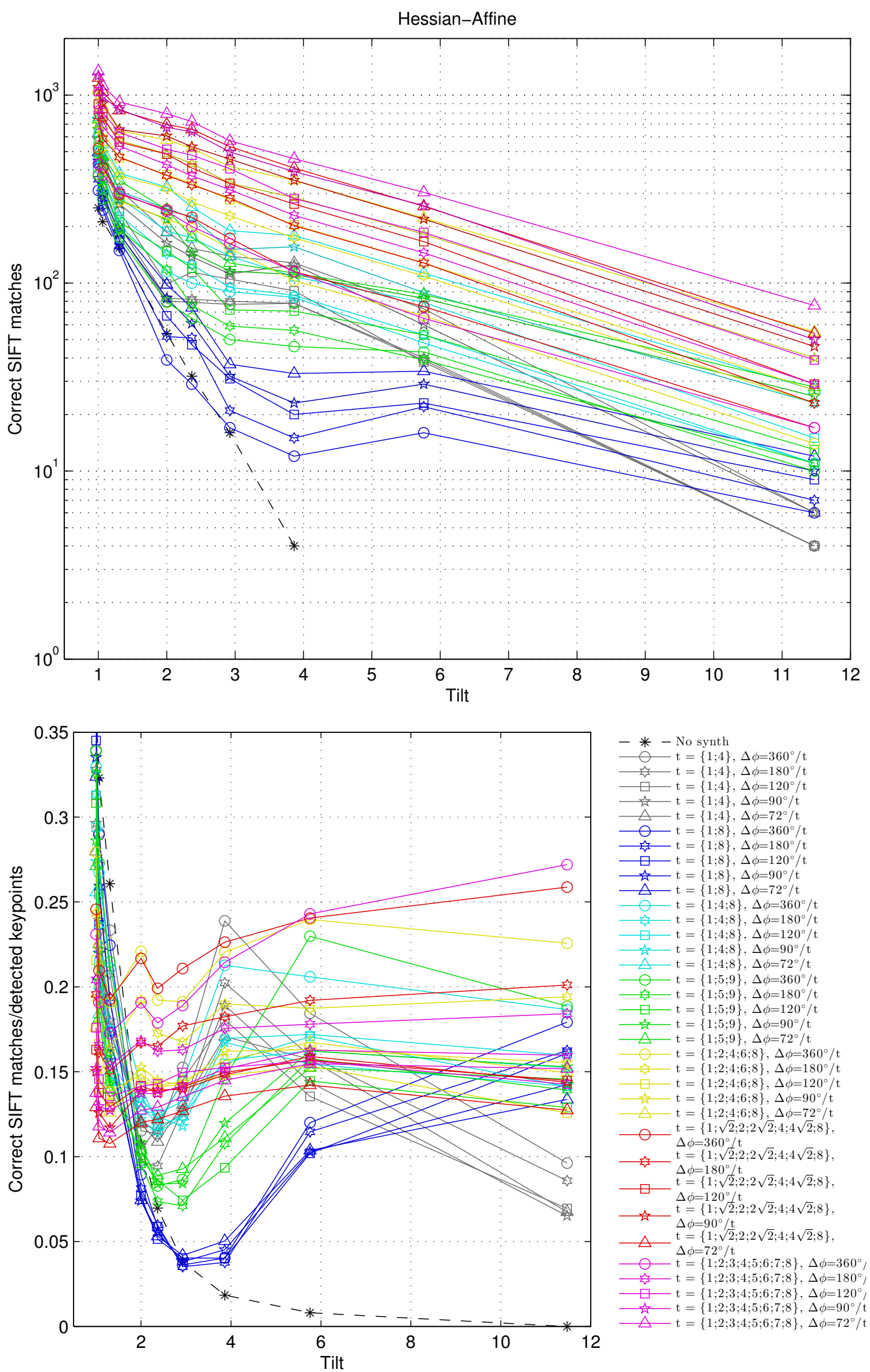

Figure 11. Comparison of Hessian-Affine view synthesis configurations on the synthetic dataset. Upper graph - the number of correct SIFT matches a robust minimum (value 4\% quantile) over 100 random images from [21]. Lower graph - the ratio of the number of correct matches to the number of detected regions; the mean over 100 random images. 

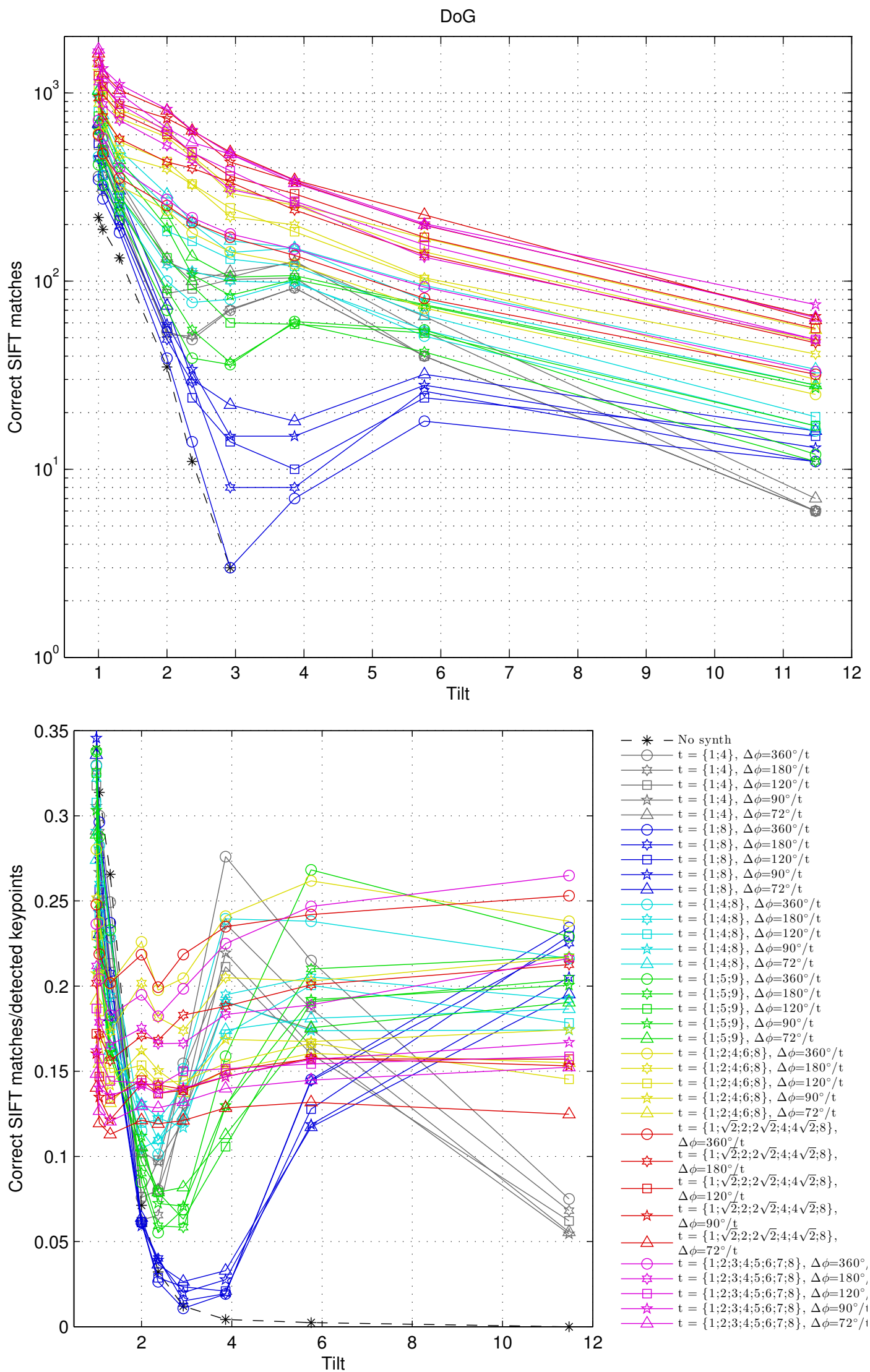

Figure 12. Comparison of DoG view synthesis configurations on the synthetic dataset. Upper graph - the number of correct SIFT matches a robust minimum (value 4\% quantile) over 100 random images from [21]. Lower graph - the ratio of the number of correct matches to the number of detected regions; the mean over 100 random images. 

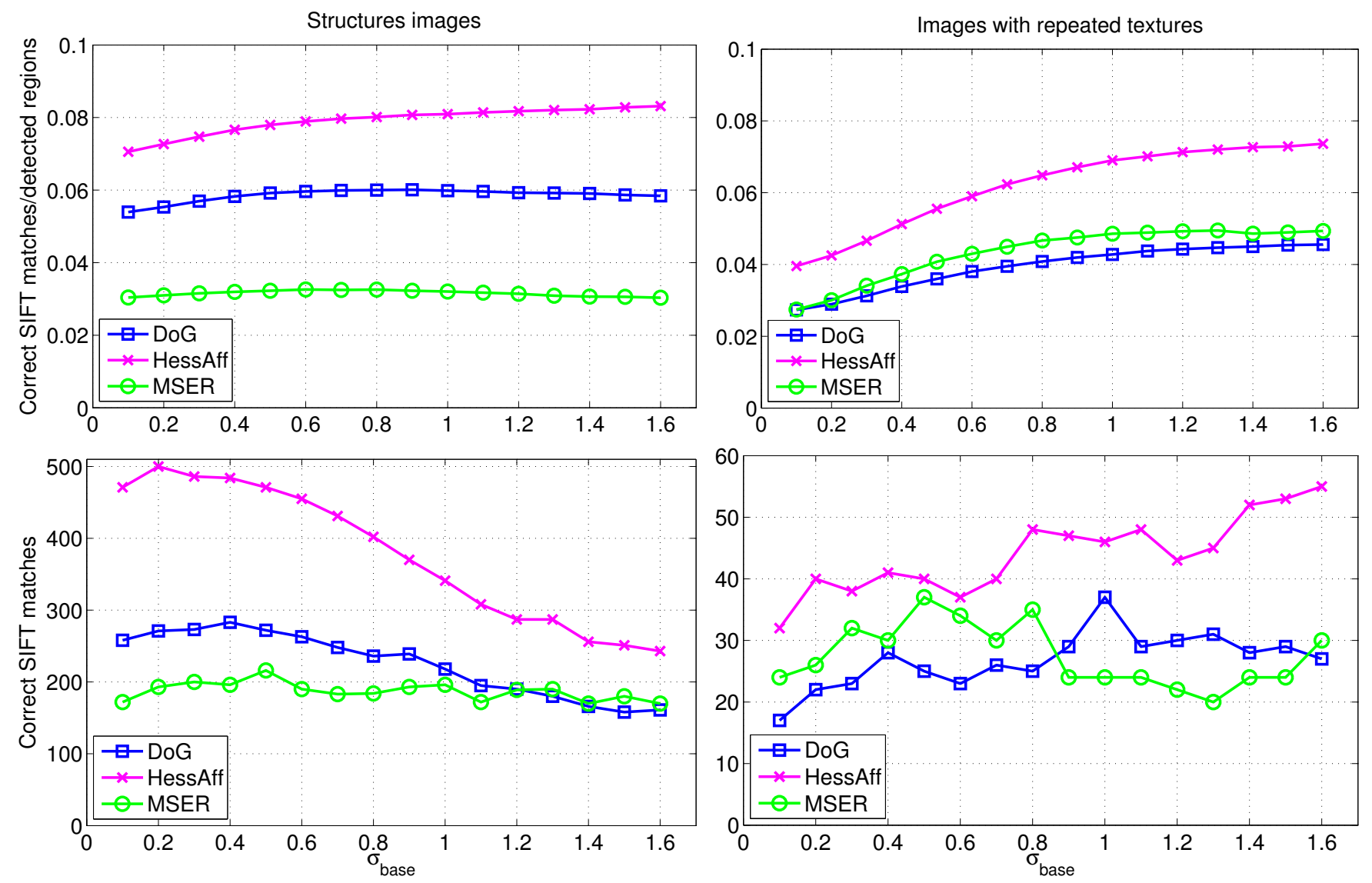

Figure 13. Matching with view synthesis (DENSE configuration) using different image pre-smoothing factor $\sigma_{b a s e}$. Rows: upper - ratio of correct SIFT matches to number of detected regions, lower - number of correct SIFT matches - robust minimum (value $4 \%$ quantile). Columns: left - structured images, right - images with repeated patterns.
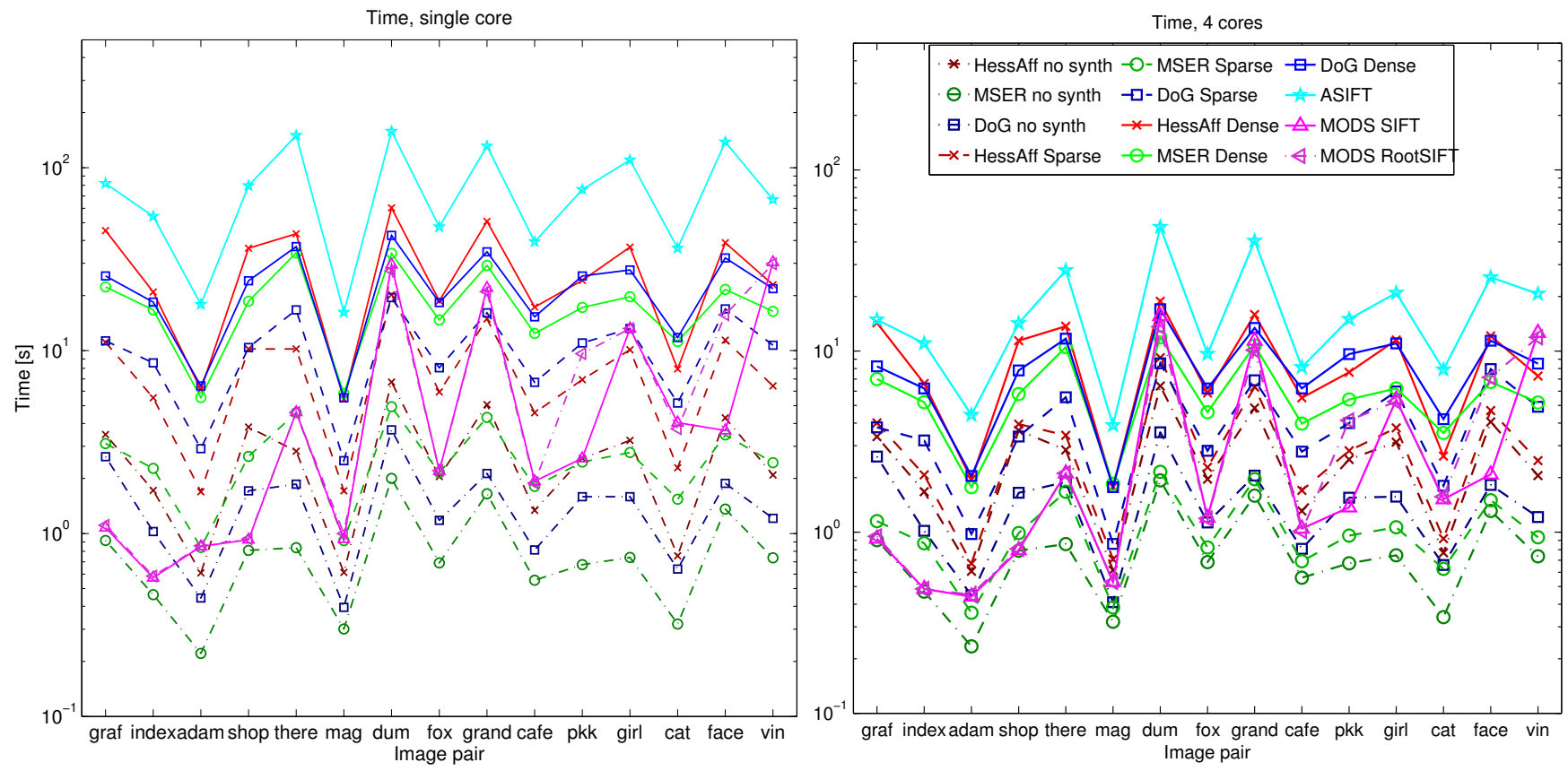

Figure 14. Running time of the different view synthesis configurations (Table 1). Left -1 core, right -4 cores. 
Table 7. Performance on the EVD dataset. MSER, no view synthesis. Results with less than 8 correct inliers are in red.

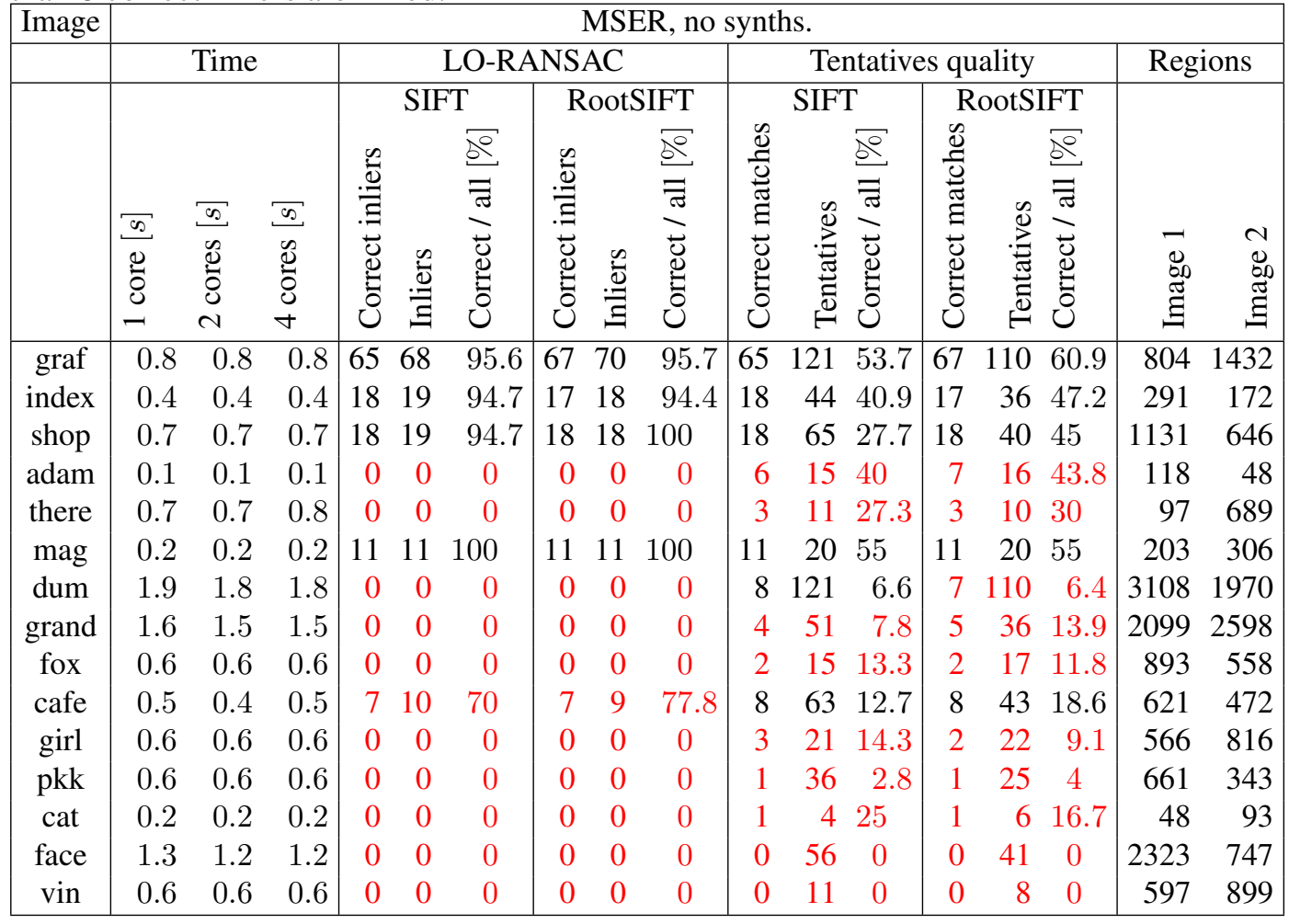

Table 8. Performance on the EVD dataset. MSER, scale view synthesis only. Results with less than 8 correct inliers are in red.

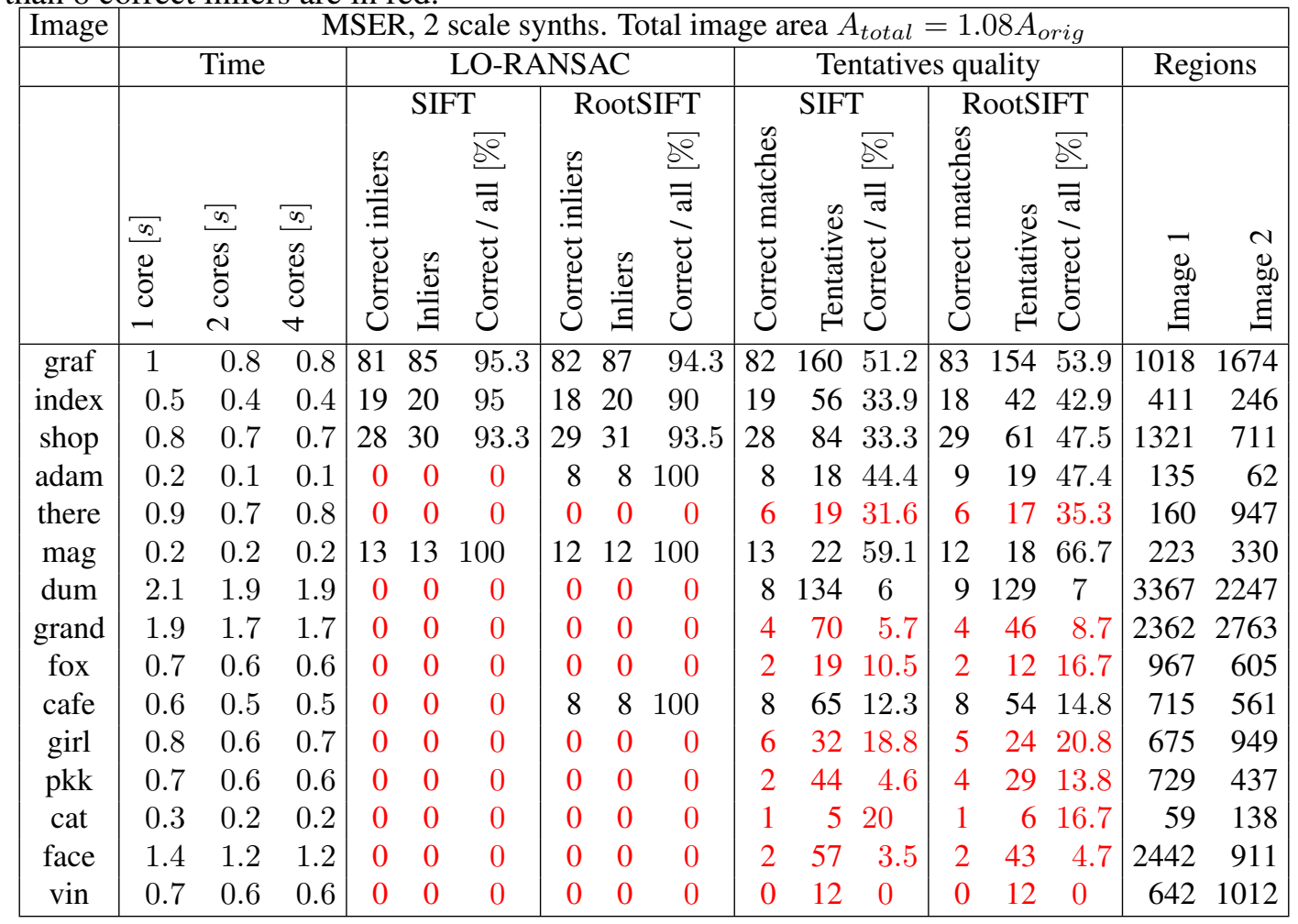


Table 9. Performance on the EVD dataset.Hessian-Affine, no view synthesis. Results with less than 8 correct inliers are in red.

\begin{tabular}{|c|c|c|c|c|c|c|c|c|c|c|c|c|c|c|c|c|c|}
\hline \multirow{4}{*}{ Image } & \multicolumn{17}{|c|}{ HessAff, no synths. } \\
\hline & \multicolumn{3}{|c|}{ Time } & \multicolumn{6}{|c|}{ LO-RANSAC } & \multicolumn{6}{|c|}{ Tentatives quality } & \multicolumn{2}{|c|}{ Regions } \\
\hline & & & & \multicolumn{3}{|c|}{ SIFT } & \multicolumn{3}{|c|}{ RootSIFT } & \multicolumn{3}{|c|}{ SIFT } & \multicolumn{3}{|c|}{ RootSIFT } & & \\
\hline & $\begin{array}{l}\infty \\
0 \\
0 \\
0 \\
0\end{array}$ & $\begin{array}{l}\infty \\
\infty \\
0 \\
0 \\
0 \\
\sim\end{array}$ & $\begin{array}{l}\infty \\
\infty \\
0 \\
0 \\
0 \\
\forall\end{array}$ & 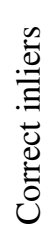 & $\stackrel{\mathscr{\Xi}}{\Xi}$ & 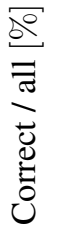 & 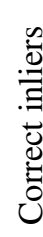 &.$\stackrel{\mathscr{\Xi}}{\Xi}$ & $\begin{array}{l}\overline{0} \\
\overline{0} \\
\overline{0} \\
\overline{0} \\
\stackrel{0}{0} \\
0 \\
0\end{array}$ & 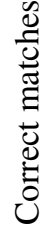 & 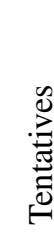 & 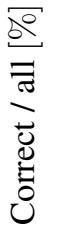 & 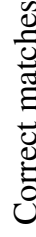 & 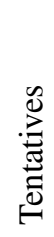 & 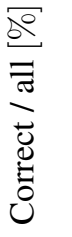 & 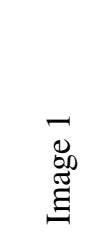 & 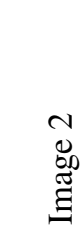 \\
\hline graf & 3.4 & 3.3 & 3.3 & 14 & 17 & 82.4 & 16 & 19 & 84.2 & 15 & 141 & 10.6 & 19 & 97 & 19.6 & 3630 & 4614 \\
\hline index & 1.6 & 1.6 & 1.6 & 0 & 0 & 0 & 0 & 0 & 0 & 0 & 114 & 0 & 1 & 79 & 1.3 & 2188 & 874 \\
\hline shop & 3.7 & 3.6 & 3.5 & 0 & 0 & 0 & 0 & 0 & 0 & 0 & 78 & 0 & 0 & 39 & 0 & 5675 & 2657 \\
\hline adam & 0.5 & 0.6 & 0.5 & 0 & 0 & 0 & 0 & 0 & 0 & 2 & 24 & 8.3 & 2 & 19 & 10.5 & 812 & 208 \\
\hline there & 2.7 & 2.7 & 2.7 & 0 & 0 & 0 & 0 & 0 & 0 & 0 & 8 & 0 & 0 & 9 & 0 & 467 & 3659 \\
\hline mag & 0.5 & 0.5 & 0.5 & 0 & 0 & 0 & 0 & 0 & 0 & 0 & 12 & 0 & 0 & 7 & 0 & 502 & 784 \\
\hline dum & 6.6 & 6.5 & 6.3 & 0 & 0 & 0 & 0 & 8 & 0 & 6 & 147 & 4.1 & & 76 & 4 & 9248 & 6666 \\
\hline grand & 5 & 4.8 & 4.8 & 0 & 0 & 0 & 0 & 0 & 0 & 3 & 62 & 4.8 & 3 & 34 & 8.8 & 6364 & 6555 \\
\hline fox & 2 & 1.9 & 1.9 & 0 & 0 & 0 & 0 & 0 & 0 & 0 & 17 & 0 & 0 & 8 & 0 & 3324 & 1393 \\
\hline cafe & 1.2 & 1.2 & 1.2 & 0 & 10 & 0 & 0 & 11 & 0 & 2 & 58 & 3.5 & 1 & 42 & 2.4 & 1510 & 1184 \\
\hline girl & 3.1 & 3.1 & 3 & 0 & 0 & 0 & 0 & 0 & 0 & 0 & 29 & 0 & 0 & 20 & 0 & 2808 & 4306 \\
\hline pkk & 2.5 & 2.4 & 2.4 & 0 & 0 & 0 & 0 & 0 & 0 & 0 & 39 & 0 & 0 & 16 & 0 & 3832 & 1568 \\
\hline cat & 0.7 & 0.7 & 0.7 & 0 & 0 & 0 & 0 & 0 & 0 & 1 & 18 & 5.6 & 0 & 13 & 0 & 388 & 581 \\
\hline face & 4.2 & 4 & 4 & 0 & 0 & 0 & 0 & 0 & 0 & 0 & 21 & 0 & 0 & 21 & 0 & 6283 & 3638 \\
\hline vin & 2 & 1.9 & 2 & 0 & 0 & 0 & 0 & 0 & 0 & 0 & 25 & 0 & 0 & 20 & 0 & 1759 & 2913 \\
\hline
\end{tabular}

Table 10. Performance on the EVD dataset. DoG, no view synthesis. Results with less than 8 correct inliers are in red.

\begin{tabular}{|c|c|c|c|c|c|c|c|c|c|c|c|c|c|c|c|c|c|}
\hline \multirow[t]{4}{*}{ Image } & \multicolumn{17}{|c|}{ DoG, no synths. } \\
\hline & \multicolumn{3}{|c|}{ Time } & \multicolumn{6}{|c|}{ LO-RANSAC } & \multicolumn{6}{|c|}{ Tentatives quality } & \multicolumn{2}{|c|}{ Regions } \\
\hline & \multirow[b]{2}{*}{$\begin{array}{l}\infty \\
0 \\
0 \\
0 \\
- \\
\end{array}$} & \multirow[b]{2}{*}{$\begin{array}{l}\tilde{\omega} \\
\tilde{u} \\
\dot{0} \\
\dot{0} \\
i\end{array}$} & \multirow[b]{2}{*}{ 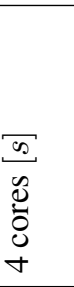 } & \multicolumn{3}{|c|}{ SIFT } & \multicolumn{3}{|c|}{ RootSIFT } & \multicolumn{3}{|c|}{ SIFT } & \multicolumn{3}{|c|}{\begin{tabular}{|l|} 
RootSIFT \\
\end{tabular}} & \multirow{2}{*}{\multicolumn{2}{|c|}{ 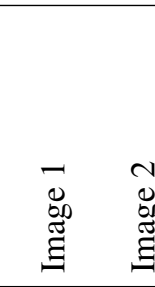 }} \\
\hline & & & & 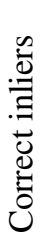 & $\stackrel{\mathscr{0}}{\stackrel{0}{\Xi}}$ & 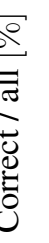 & 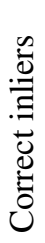 & & & ర্ & & 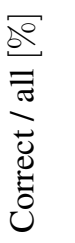 & $n$ & & 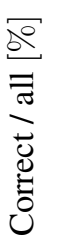 & & \\
\hline graf & 2.5 & 2.5 & 2.5 & 0 & 0 & 0 & 0 & 0 & 0 & 3 & 120 & 2.5 & & 83 & 4.8 & 1682 & 2419 \\
\hline index & 0.9 & 0.9 & 0.9 & 0 & 0 & 0 & 0 & 0 & 0 & 0 & 106 & 0 & 0 & 80 & 0 & 1171 & 516 \\
\hline shop & 1.6 & 1.6 & 1.6 & 0 & 0 & 0 & 0 & 0 & 0 & 0 & 93 & 0 & & 83 & 0 & 2570 & 1238 \\
\hline adam & 0.3 & 0.3 & 0.3 & 0 & 0 & 0 & 0 & 0 & 0 & 0 & 31 & 0 & & 29 & 0 & 495 & 132 \\
\hline there & 1.8 & 1.9 & 1.8 & 0 & 0 & 0 & 0 & 0 & 0 & 0 & 49 & 0 & & 30 & 0 & 541 & 2476 \\
\hline mag & 0.3 & 0.3 & 0.3 & 0 & 0 & 0 & 0 & 0 & 0 & 0 & 17 & 0 & 0 & 15 & 0 & 252 & 370 \\
\hline dum & 3.6 & 3.5 & 3.5 & 0 & 0 & 0 & 0 & 0 & 0 & 0 & 144 & 0 & & 89 & 0 & 4242 & 2791 \\
\hline grand & 2 & 2 & 2 & 0 & 0 & 0 & 0 & 0 & 0 & 0 & 66 & 0 & 1 & 47 & 2.1 & 2754 & 2956 \\
\hline fox & 1.1 & 1.1 & 1 & 0 & 0 & 0 & 0 & 0 & 0 & 0 & 42 & 0 & & 22 & 0 & 1764 & 817 \\
\hline cafe & 0.7 & 0.7 & 0.7 & 0 & 10 & 0 & 0 & 8 & 0 & 1 & 60 & 1.7 & & 46 & 0 & 847 & 813 \\
\hline girl & 1.5 & 1.5 & 1.5 & 0 & 0 & 0 & 0 & 0 & 0 & 0 & 60 & 0 & 0 & 39 & 0 & 1217 & 2190 \\
\hline pkk & 1.5 & 1.5 & 1.5 & 0 & 0 & 0 & 0 & 0 & 0 & 0 & 40 & 0 & 0 & 26 & 0 & 2091 & 1487 \\
\hline cat & 0.5 & 0.5 & 0.6 & 0 & 0 & 0 & 0 & 0 & 0 & 0 & 30 & 0 & & 19 & 0 & 262 & 519 \\
\hline face & 1.8 & 1.8 & 1.7 & 0 & 0 & 0 & 0 & 0 & 0 & 0 & 34 & 0 & 0 & 39 & 0 & 2406 & 2457 \\
\hline vin & 1.1 & 1.2 & 1.1 & 0 & 0 & 0 & 0 & 0 & 0 & 0 & 48 & 0 & & 32 & 0 & 876 & 1661 \\
\hline
\end{tabular}


Table 11. Performance on the EVD dataset.MSER, SPARSE configuration. Results with less than 8 correct inliers are in red.

\begin{tabular}{|c|c|c|c|c|c|c|c|c|c|c|c|c|c|c|c|c|c|}
\hline \multirow[t]{4}{*}{ Image } & \multicolumn{17}{|c|}{$\begin{array}{l}\text { MSER, } 6 \text { tilt synths } \mathrm{x}(1+2 \text { scale synth }) . \Delta \phi=360^{\circ} / t, \\
\quad \mathrm{t}=\{1 ; 5 ; 9\} . \text { Total image area } A_{\text {total }}=2.8 A_{\text {orig }}\end{array}$} \\
\hline & \multicolumn{3}{|c|}{ Time } & \multicolumn{6}{|c|}{ LO-RANSAC } & \multicolumn{6}{|c|}{ Tentatives quality } & \multicolumn{2}{|c|}{ Regions } \\
\hline & & & & \multicolumn{3}{|c|}{ SIFT } & \multicolumn{3}{|c|}{ RootSIFT } & \multicolumn{3}{|c|}{ SIFT } & \multicolumn{3}{|c|}{ RootSIFT } & & \\
\hline & $\begin{array}{l}\frac{\rho}{0} \\
\tilde{0} \\
0\end{array}$ & $\begin{array}{l}\omega \\
\infty \\
0 \\
0 \\
0 \\
N\end{array}$ & 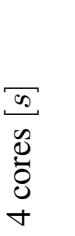 & 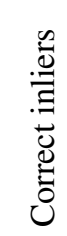 & $\stackrel{\stackrel{0}{0}}{.}$ & 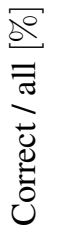 & 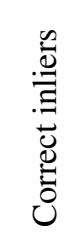 & 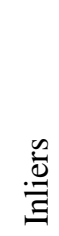 & $\begin{array}{l}\overline{s e} \\
\bar{\sigma} \\
\bar{d} \\
\stackrel{0}{0} \\
0\end{array}$ & 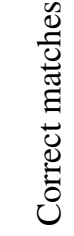 & 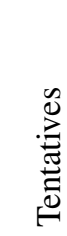 & 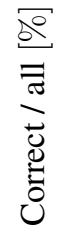 & 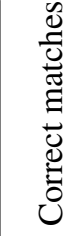 & 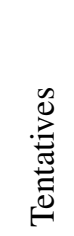 & $\begin{array}{l}\overline{0} \\
\bar{\sigma} \\
\overline{0} \\
0 \\
0 \\
0\end{array}$ & $\begin{array}{l}\bar{\Xi} \\
8 \\
\tilde{\Xi} \\
\bar{\Xi}\end{array}$ & 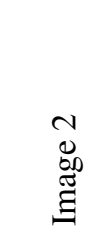 \\
\hline graf & 3 & 1.6 & 1.1 & 167 & 173 & 96.5 & 165 & 169 & 97.6 & 175 & 340 & 51.5 & 170 & 339 & 50.1 & 2780 & 3782 \\
\hline index & 2.2 & 1.2 & 0.8 & 23 & 32 & 71.9 & 24 & 35 & 68.6 & 25 & 106 & 23.6 & 27 & 103 & 26.2 & 1204 & 736 \\
\hline shop & 2.5 & 1.4 & 0.9 & 67 & 69 & 97.1 & 73 & 74 & 98.6 & 67 & 172 & 39 & 73 & 163 & 44.8 & 2899 & 1474 \\
\hline adam & 0.7 & 0.4 & 0.3 & 18 & 20 & 90 & 18 & 21 & 85.7 & 20 & 48 & 41.7 & 20 & 42 & 47.6 & 357 & 164 \\
\hline there & 4.5 & 2.5 & 1.6 & 12 & 19 & 63.2 & 12 & 18 & 66.7 & 15 & 65 & 23.1 & 17 & 61 & 27.9 & 571 & 2833 \\
\hline & 0.8 & 0.5 & 0.3 & 25 & 27 & 92.6 & 28 & 28 & 100 & 26 & 47 & 55.3 & 28 & 40 & 70 & 393 & 509 \\
\hline $\mathrm{du}$ & 4.8 & 2.7 & 2.1 & 0 & 0 & 0 & 0 & 0 & 0 & 12 & 229 & 5.2 & 14 & 173 & 8.1 & 6276 & 4579 \\
\hline grand & 4.2 & 2.4 & 1.9 & 0 & 0 & 0 & 9 & 14 & 64.3 & 10 & 163 & 6.1 & 9 & 105 & 8.6 & 4840 & 4346 \\
\hline for & 2.1 & 1.1 & 0.7 & 12 & 17 & 70.6 & 19 & 20 & 95 & 16 & 68 & 23.5 & 20 & 61 & 32.8 & 1717 & 1011 \\
\hline cafe & 1.7 & 1 & 0. & 13 & 20 & 65 & 14 & 20 & 70 & 15 & 117 & 12.8 & 16 & 104 & 15.4 & 1402 & 1319 \\
\hline gir & 2. & 1.5 & 1 & 10 & 15 & 66.7 & 0 & 0 & 0 & 11 & 82 & 13.4 & 10 & 61 & 16.4 & 1479 & 2208 \\
\hline $\mathrm{pk}$ & 2.4 & 1.3 & 0.9 & 4 & 14 & 28.6 & 6 & 10 & 60 & 7 & 68 & 10.3 & 8 & 45 & 17.8 & 1229 & 1267 \\
\hline $\mathrm{ca}$ & 1.4 & 0.8 & 0. & 0 & 0 & 0 & 0 & 0 & 0 & 2 & 13 & 15.4 & 2 & 13 & 15.4 & 144 & 440 \\
\hline face & 3.4 & 1.9 & 1. & 0 & 0 & 0 & 9 & 14 & 64.3 & 11 & 93 & 11.8 & 10 & 86 & 11.6 & 3411 & 2371 \\
\hline vin & 2.3 & 1.3 & 0.8 & 0 & 0 & 0 & 0 & 0 & 0 & 4 & 24 & 16.7 & 4 & 21 & 19 & 1106 & 1881 \\
\hline
\end{tabular}

Table 12. Performance on the EVD dataset.Hessian-Affine, SPARSE configuration. Results with less than 8 correct inliers are in red.

\begin{tabular}{|c|c|c|c|c|c|c|c|c|c|c|c|c|c|c|c|c|c|}
\hline \multirow[t]{4}{*}{ Image } & \multicolumn{17}{|c|}{$\begin{array}{c}\text { HessAff, } 10 \text { synths. } \Delta \phi=360^{\circ} / t \\
\mathrm{t}=\{1 ; \sqrt{2} ; 2 ; 2 \sqrt{2} ; 4 ; 4 \sqrt{2} ; 8\} . \text { Total image area } A_{\text {total }}=4 A_{\text {orig }}\end{array}$} \\
\hline & \multicolumn{3}{|c|}{ Time } & \multicolumn{6}{|c|}{ LO-RANSAC } & \multicolumn{6}{|c|}{ Tentatives quality } & \multicolumn{2}{|c|}{ Regions } \\
\hline & \multirow[b]{2}{*}{ 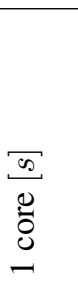 } & \multirow[b]{2}{*}{ 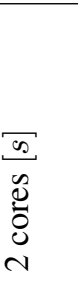 } & \multirow[b]{2}{*}{$\begin{array}{l}\tilde{\sigma} \\
\tilde{0} \\
\dot{0} \\
\dot{\theta} \\
\dot{\sigma}\end{array}$} & \multicolumn{3}{|c|}{ SIFT } & \multicolumn{3}{|c|}{ RootSIFT } & \multicolumn{3}{|c|}{ SIFT } & \multicolumn{3}{|c|}{ RootSIFT } & & \\
\hline & & & & 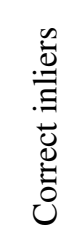 & $\stackrel{\vec{\Xi}}{\Xi}$ & 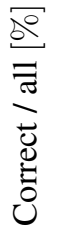 & 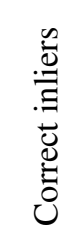 & $\stackrel{\mathscr{U}}{\Xi}$ & 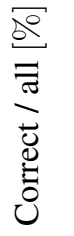 & 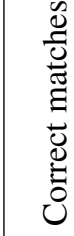 & 氖 & 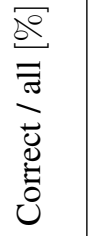 & 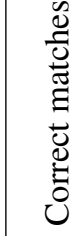 & & 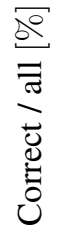 & & $\underset{\Xi}{\mathscr{E}}$ \\
\hline graf & 11 & 5.9 & 3.9 & 371 & 379 & 97.9 & 375 & 383 & 97.9 & 375 & 799 & 46.9 & 384 & 765 & 50.2 & 12519 & 14144 \\
\hline index & 5.4 & 3 & 2 & 23 & 40 & 57.5 & 34 & 52 & 65.4 & 34 & 412 & 8.3 & 44 & 284 & 15 & 67 & \\
\hline shop & .1 & 5.4 & 3.9 & 34 & 143 & 93.7 & 133 & 141 & 94.3 & 138 & 341 & 40.5 & 135 & 257 & 52.5 & & \\
\hline adam & 1. & 0.8 & 06 & 86 & 93 & 92.5 & 86 & 99 & 86 & 88 & 157 & 56.1 & 88 & 151 & 5 & 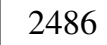 & 63 \\
\hline there & 10.1 & 5.3 & 3.8 & 58 & 66 & 87.9 & 49 & 56 & 87. & 64 & 223 & 28.7 & 52 & 163 & 31.9 & 2702 & 15991 \\
\hline mag & 1. & 0.9 & 0.6 & 55 & 60 & 91.7 & 54 & 59 & 91.5 & 57 & 95 & 60 & 57 & 93 & 61.3 & 1664 & 2162 \\
\hline dum & 20.1 & 11.7 & 9.1 & 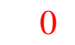 & O & 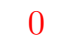 & 10 & 12 & 83.3 & 10 & 254 & 3.9 & 11 & 150 & 7.3 & 66 & 19132 \\
\hline grand & 14.8 & 8.1 & 6.2 & 0 & 0 & 0 & 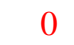 & 0 & 0 & 8 & 152 & 5.3 & 5 & 65 & 7.7 & 991 & 1595 \\
\hline & & 3. & 2. & 27 & 34 & 79.4 & 22 & 32 & 68.8 & 30 & 99 & 30.3 & 27 & 72 & 37.5 & 27 & \\
\hline $\mathrm{cal}$ & 1 & 2. & & 0 & 14 & 0 & 0 & 14 & U & 9 & 135 & 6.7 & & 112 & 6.3 & 42 & \\
\hline gi & 10 & 5. & & 16 & 25 & 64 & 4 & 23 & 60. & 18 & 170 & 10.6 & 17 & 120 & 14.2 & 981 & 1389 \\
\hline pkk & 6 & 3.7 & 2. & 21 & 25 & 84 & 12 & 19 & 63.2 & 25 & 105 & 23.8 & 17 & 84 & 20.2 & 457 & 5818 \\
\hline cat & 2.2 & 1.2 & 0.8 & 24 & 26 & 92.3 & 21 & 29 & 72.4 & 24 & 75 & 32 & 23 & 67 & 34.3 & 1118 & 2839 \\
\hline face & 11.3 & 6 & 4.6 & 35 & 39 & 89.7 & 17 & 20 & 85 & 38 & 139 & 27.3 & 18 & 93 & 19.4 & 15446 & 10900 \\
\hline vin & 6.3 & 3.4 & 2.4 & 0 & 0 & 0 & P & & 0 & 0 & 46 & 0 & 0 & 31 & 0 & 5656 & 8401 \\
\hline
\end{tabular}


Table 13. Performance on the EVD dataset.DoG, SPARSE configuration. Results with less than 8 correct inliers are in red.

\begin{tabular}{|c|c|c|c|c|c|c|c|c|c|c|c|c|c|c|c|c|c|}
\hline \multirow[t]{4}{*}{ Image } & \multicolumn{17}{|c|}{$\begin{array}{c}\text { DoG, } 30 \text { synths. } \Delta \phi=120^{\circ} / t \\
\mathrm{t}=\{1 ; 2 ; 4 ; 6 ; 8\}, \text { Total image area } A_{\text {total }}=7 A_{\text {orig }}\end{array}$} \\
\hline & \multicolumn{3}{|c|}{ Time } & \multicolumn{6}{|c|}{ LO-RANSAC } & \multicolumn{6}{|c|}{ Tentatives quality } & \multicolumn{2}{|c|}{ Regions } \\
\hline & & & & \multicolumn{3}{|c|}{ SIFT } & \multicolumn{3}{|c|}{ RootSIFT } & \multicolumn{3}{|c|}{ SIFT } & \multicolumn{3}{|c|}{ RootSIFT } & & \\
\hline & $\begin{array}{l}\omega \\
\tilde{0} \\
\tilde{0} \\
-\end{array}$ & 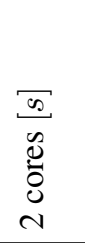 & $\begin{array}{l}\infty \\
\infty \\
0 \\
0 \\
0 \\
\dot{0}\end{array}$ & 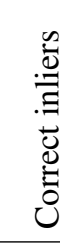 & 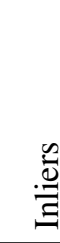 & 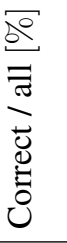 & 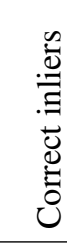 & $\stackrel{\mathscr{U}}{\Xi}$ & 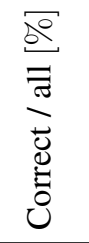 & 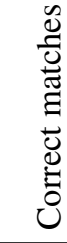 & 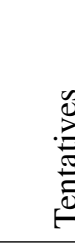 & 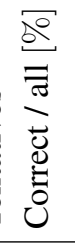 & 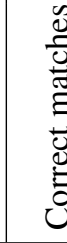 & the & 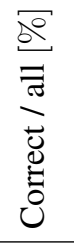 & $\begin{array}{l}\mathbb{\Xi} \\
\Xi \\
\Xi\end{array}$ & $\begin{array}{l}\tilde{\Xi} \\
\tilde{\Xi} \\
\tilde{\Xi}\end{array}$ \\
\hline graf & 11.2 & 6 & 3.7 & 354 & 368 & 96.2 & 363 & 376 & 96.5 & 368 & 1121 & 32.8 & 379 & 1019 & 37.2 & 12708 & 17175 \\
\hline index & 8.5 & 4.8 & 3.1 & 52 & 67 & 77.6 & 51 & 73 & 69.9 & 63 & 930 & 6.8 & 67 & 692 & 9.7 & 7986 & 4770 \\
\hline shop & 10.3 & 5.5 & 3.3 & 62 & 65 & 95.4 & 66 & 72 & 91.7 & 63 & 575 & 11 & 69 & 374 & 18.4 & 15747 & 7873 \\
\hline adam & 2.8 & 1.5 & 0.9 & 70 & 72 & 97.2 & 69 & 79 & 87.3 & 74 & 283 & 26.1 & 75 & 241 & 31.1 & 3181 & 895 \\
\hline there & 16.6 & 9 & 5.5 & 37 & 51 & 72.5 & 25 & 42 & 59.5 & 43 & 533 & 8.1 & 33 & 365 & 9 & 5389 & 21491 \\
\hline mag & 2.4 & 1.3 & 0.8 & 37 & 39 & 94.9 & 38 & 38 & 100 & 38 & 110 & 34.5 & 40 & 94 & 42.6 & 1467 & 1678 \\
\hline dum & 19.4 & 12 & 8.5 & 13 & 20 & 65 & 16 & 22 & 72.7 & 15 & 646 & 2.3 & 17 & 455 & 3.7 & 23607 & 19402 \\
\hline grand & 16 & 9.7 & 6.8 & 0 & 0 & 0 & 16 & 19 & 84.2 & 11 & 447 & 2.5 & 16 & 256 & 6.3 & 18270 & 15387 \\
\hline fox & 8 & 4.4 & 2.7 & 14 & 21 & 66.7 & 11 & 21 & 52.4 & 15 & 278 & 5.4 & 12 & 157 & 7.6 & 10026 & 4995 \\
\hline cafe & 6.6 & 3.9 & 2.7 & 11 & 17 & 64.7 & 13 & 19 & 68.4 & 12 & 271 & 4.4 & 15 & 207 & 7.3 & 5153 & 6541 \\
\hline girl & 13.1 & 8.2 & 5.9 & 0 & 0 & 0 & 0 & 0 & 0 & 6 & 370 & 1.6 & 7 & 226 & 3.1 & 7745 & 14677 \\
\hline pkk & 10.9 & 6.1 & 3. & 0 & 0 & 0 & 0 & 0 & 0 & 8 & 358 & 2.2 & 7 & 238 & 2.9 & 9934 & 10704 \\
\hline cat & 5.1 & 2.8 & 1. & 0 & 0 & 0 & 2 & 9 & 22.2 & 2 & 119 & 1.7 & 4 & 79 & 5.1 & 1815 & 3750 \\
\hline face & 16.8 & 10.6 & 7.8 & 0 & 0 & 0 & 0 & 0 & 0 & 11 & 584 & 1.9 & 5 & 396 & 1.3 & 17217 & 14204 \\
\hline vin & 10.6 & 6.7 & 4.8 & 0 & 0 & 0 & 0 & 0 & 0 & 6 & 250 & 2.4 & 2 & 131 & 1.5 & 6470 & 9861 \\
\hline
\end{tabular}

Table 14. Performance on the EVD dataset. MSER,DENSE configuration. Results with less than 8 correct inliers are in red.

\begin{tabular}{|c|c|c|c|c|c|c|c|c|c|c|c|c|c|c|c|c|c|}
\hline \multirow[t]{4}{*}{ Image } & \multicolumn{17}{|c|}{$\begin{array}{l}\text { MSER, } 14 \text { tilt synths } \mathrm{x}\left(1+2 \text { scale synth). } \Delta \phi=180^{\circ} / t,\right. \\
\quad \mathrm{t}=\{1 ; 5 ; 9\} . \text { Total image area } A_{\text {total }}=4.2 A_{\text {orig }}\end{array}$} \\
\hline & \multicolumn{3}{|c|}{ Time } & \multicolumn{6}{|c|}{ LO-RANSAC } & \multicolumn{6}{|c|}{ Tentatives quality } & \multicolumn{2}{|c|}{ Regions } \\
\hline & \multirow[b]{2}{*}{ 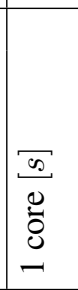 } & \multirow[b]{2}{*}{ 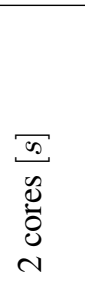 } & \multirow[b]{2}{*}{ 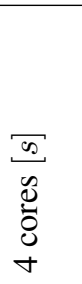 } & \multicolumn{3}{|c|}{ SIFT } & \multicolumn{3}{|c|}{ RootSIFT } & \multicolumn{3}{|c|}{ SIFT } & \multicolumn{3}{|c|}{ RootSIFT } & \multirow[b]{2}{*}{ 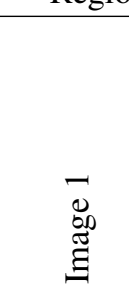 } & \multirow[b]{2}{*}{$\begin{array}{l}N \\
0 \\
\Xi \\
\Xi\end{array}$} \\
\hline & & & & 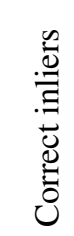 & 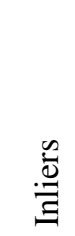 & 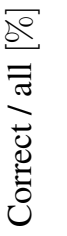 & 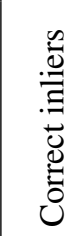 & $\stackrel{\square}{\Xi}$ & 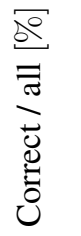 & 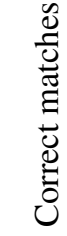 & & $\begin{array}{l}\frac{\overline{0}}{\bar{\sigma}} \\
\overline{\bar{\sigma}} \\
\overline{0} \\
\stackrel{0}{0}\end{array}$ & 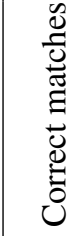 & 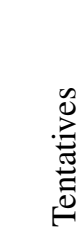 & 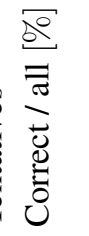 & & \\
\hline graf & 22.2 & 11.7 & 6.9 & 330 & 361 & $\overline{91.4}$ & 332 & 353 & 94.1 & 339 & 1061 & 32 & 342 & 1014 & 33.7 & 20454 & 26177 \\
\hline index & 16.6 & 8.8 & 5.1 & 51 & 65 & 78.5 & 56 & 76 & 73.7 & 60 & 390 & 15.4 & 66 & 391 & 16.9 & 9087 & 5733 \\
\hline shop & 18.5 & 9.7 & 5.7 & 139 & 152 & 91.4 & 147 & 161 & 91.3 & 145 & 530 & 27.4 & 150 & 446 & 33.6 & 19560 & 9905 \\
\hline adam & 5.4 & 2.8 & 1.7 & 36 & 41 & 87.8 & 26 & 38 & 68.4 & 38 & 114 & 33.3 & 34 & 104 & 32.7 & 2161 & 1201 \\
\hline there & 34.1 & 18 & 10.4 & 43 & 68 & 63.2 & 46 & 69 & 66.7 & 59 & 243 & 24.3 & 61 & 247 & 24.7 & 4405 & 20824 \\
\hline $\mathrm{mag}$ & 5.7 & 3 & 1.8 & 30 & 36 & 83.3 & 32 & 39 & 82.1 & 35 & 114 & 30.7 & 35 & 105 & 33.3 & 2123 & 2836 \\
\hline dum & 33.9 & 18.7 & 11.8 & 42 & 47 & 89.4 & 34 & 39 & 87.2 & 43 & 867 & 5 & 38 & 653 & 5.8 & 38881 & 29687 \\
\hline grand & 29.1 & 16.5 & 10.6 & 0 & 0 & 0 & 0 & 0 & 0 & 15 & 540 & 2.8 & 12 & 349 & & 29894 & 23431 \\
\hline fox & 14.6 & 7.6 & 4.5 & 37 & 41 & 90.2 & 39 & 43 & 90.7 & 42 & 241 & 17.4 & 41 & 209 & 19.6 & 10731 & 5960 \\
\hline cafe & 12.3 & 6.5 & 3.9 & 17 & 30 & 56.7 & 19 & 32 & 59.4 & 20 & 287 & 7 & 22 & 263 & 8.4 & 8932 & 8805 \\
\hline girl & 19.6 & 10.4 & 6.1 & 9 & 25 & 36 & 11 & 21 & 52.4 & 23 & 237 & 9.7 & 16 & 192 & 8.3 & 9313 & 15567 \\
\hline pkk & 17.1 & 9.1 & 5.3 & 2 & 25 & 8 & 7 & 29 & 24.1 & 12 & 182 & 6.6 & 18 & 194 & 9.3 & 6922 & 9210 \\
\hline cat & 11.1 & 5.9 & 3.4 & U & 0 & 0 & 0 & 0 & 0 & 4 & 31 & 12.9 & 3 & 41 & 7.3 & 1084 & 3333 \\
\hline face & 21.5 & 11.3 & 6.6 & 52 & 68 & 76.5 & 55 & 70 & 78.6 & 56 & 438 & 12.8 & 64 & 358 & 17.9 & 13733 & 17135 \\
\hline vin & 16.3 & 8.6 & 5.1 & 10 & 15 & 66.7 & 11 & 17 & 64.7 & 10 & 125 & 8 & 11 & 101 & 10.9 & 6423 & 10539 \\
\hline
\end{tabular}


Table 15. Performance on the EVD dataset. Hessian-Affine, DENSE configuration. Results with less than 8 correct inliers are in red.

\begin{tabular}{|c|c|c|c|c|c|c|c|c|c|c|c|c|c|c|c|c|c|}
\hline \multirow[t]{4}{*}{ Image } & \multicolumn{17}{|c|}{$\begin{array}{c}\text { HessAff, } 50 \text { synths. } \Delta \phi=72^{\circ} / t \\
\mathrm{t}=\{1 ; 2 ; 4 ; 6 ; 8\} . \text { Total image area } A_{\text {total }}=11 A_{\text {orig }}\end{array}$} \\
\hline & \multicolumn{3}{|c|}{ Time } & \multicolumn{6}{|c|}{ LO-RANSAC } & \multicolumn{6}{|c|}{ Tentatives quality } & \multicolumn{2}{|c|}{ Regions } \\
\hline & \multirow[b]{2}{*}{$\begin{array}{l}\infty \\
0 \\
0 \\
0 \\
0 \\
-\end{array}$} & \multirow[b]{2}{*}{ 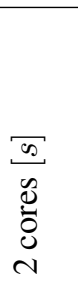 } & \multirow[b]{2}{*}{ 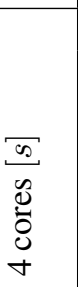 } & \multicolumn{3}{|c|}{ SIFT } & \multicolumn{3}{|c|}{ RootSIFT } & \multicolumn{3}{|c|}{ SIFT } & \multicolumn{3}{|c|}{ RootSIFT } & & \\
\hline & & & & 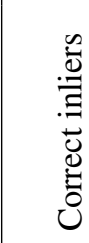 & & 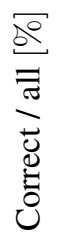 & 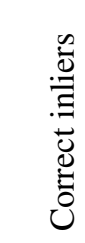 & & $\begin{array}{l}\overline{0} \\
\bar{\sigma} \\
\bar{\sigma} \\
\overline{0} \\
\stackrel{0}{0} \\
0 \\
0\end{array}$ & 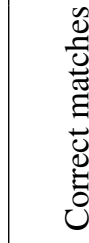 & & 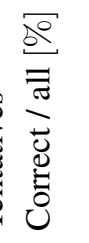 & 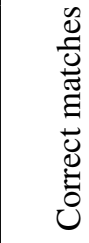 & & 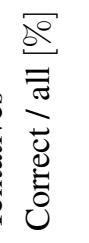 & $\begin{array}{l}\overline{\mathbb{D}} \\
\mathbb{\Xi} \\
\tilde{\xi}\end{array}$ & 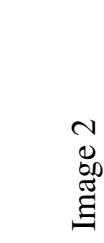 \\
\hline graf & 45.2 & 23.8 & 14.2 & \begin{tabular}{|l}
1214 \\
\end{tabular} & 1260 & 96.3 & 1235 & 1274 & 96.9 & 1249 & 2958 & 42.2 & $\mid 1267$ & 2887 & 43.9 & 46997 & 58020 \\
\hline index & 20.8 & 10.9 & 6.5 & 270 & 297 & 90.9 & 264 & 302 & 87.4 & 312 & 1698 & 18.4 & 326 & 1297 & 25.1 & 27910 & 11497 \\
\hline shop & 36.2 & 19.2 & 11.3 & 303 & 315 & 96.2 & 311 & 322 & 96.6 & 311 & 847 & 36.7 & 326 & 676 & 48.2 & 55508 & 25538 \\
\hline adam & 6 & 3.1 & 1.9 & 205 & 239 & 85.8 & 214 & 231 & 92.6 & 242 & 525 & 46.1 & 239 & 497 & 48.1 & 7616 & 2310 \\
\hline there & 43.4 & 23 & 13.6 & 211 & 274 & 77 & 189 & 234 & 80.8 & 240 & 905 & 26.5 & 212 & 680 & 31.2 & 11784 & 61930 \\
\hline mag & 5.3 & 2.8 & 1.7 & 71 & 79 & 89.9 & 72 & 76 & 94.7 & 74 & 184 & 40.2 & 73 & 151 & 48.3 & 4362 & 6296 \\
\hline dum & 60.2 & 31.6 & 18.7 & 61 & 68 & 89.7 & 66 & 74 & 89.2 & 63 & 617 & 10.2 & 68 & 419 & 16.2 & 79499 & 64321 \\
\hline grand & 50.8 & 26.6 & 15.8 & 54 & 61 & 88.5 & 42 & 54 & 77.8 & 56 & 525 & 10.7 & 46 & 276 & 16.7 & 63962 & 52899 \\
\hline fox & 18.6 & 9.7 & 5.8 & 75 & 86 & 87.2 & 74 & 84 & 88.1 & 79 & 258 & 30.6 & 76 & 205 & 37.1 & 26946 & 12327 \\
\hline cafe & 17.2 & 9.2 & 5.4 & 34 & 45 & 75.6 & 45 & 53 & 84.9 & 39 & 437 & 8.9 & 48 & 409 & 11.7 & 16538 & 18329 \\
\hline girl & 36.7 & 19.3 & 11.4 & 55 & 65 & 84.6 & 59 & 69 & 85.5 & 64 & 452 & 14.2 & 65 & 291 & 22.3 & 26776 & 49353 \\
\hline pkk & 24.1 & 12.7 & 7.5 & 40 & 73 & 54.8 & 41 & 73 & 56.2 & 52 & 349 & 14.9 & 52 & 247 & 21.1 & 25266 & 22414 \\
\hline cat & 7.8 & 4.2 & 2.5 & 21 & 38 & 55.3 & 18 & 34 & 52.9 & 37 & 147 & 25.2 & 29 & 115 & 25.2 & 3645 & 7267 \\
\hline face & 38.8 & 20.5 & 12 & 52 & 55 & 94.5 & 24 & 25 & 96 & 56 & 417 & 13.4 & 26 & 277 & 9.4 & 42689 & 38507 \\
\hline vin & 22.8 & 12.2 & 7.2 & 8 & 16 & 50 & 6 & 12 & & 10 & 147 & & & & & 16608 & 28275 \\
\hline
\end{tabular}

Table 16. Performance on the EVD dataset. DoG,DENSE configuration. Results with less than 8 correct inliers are in red.

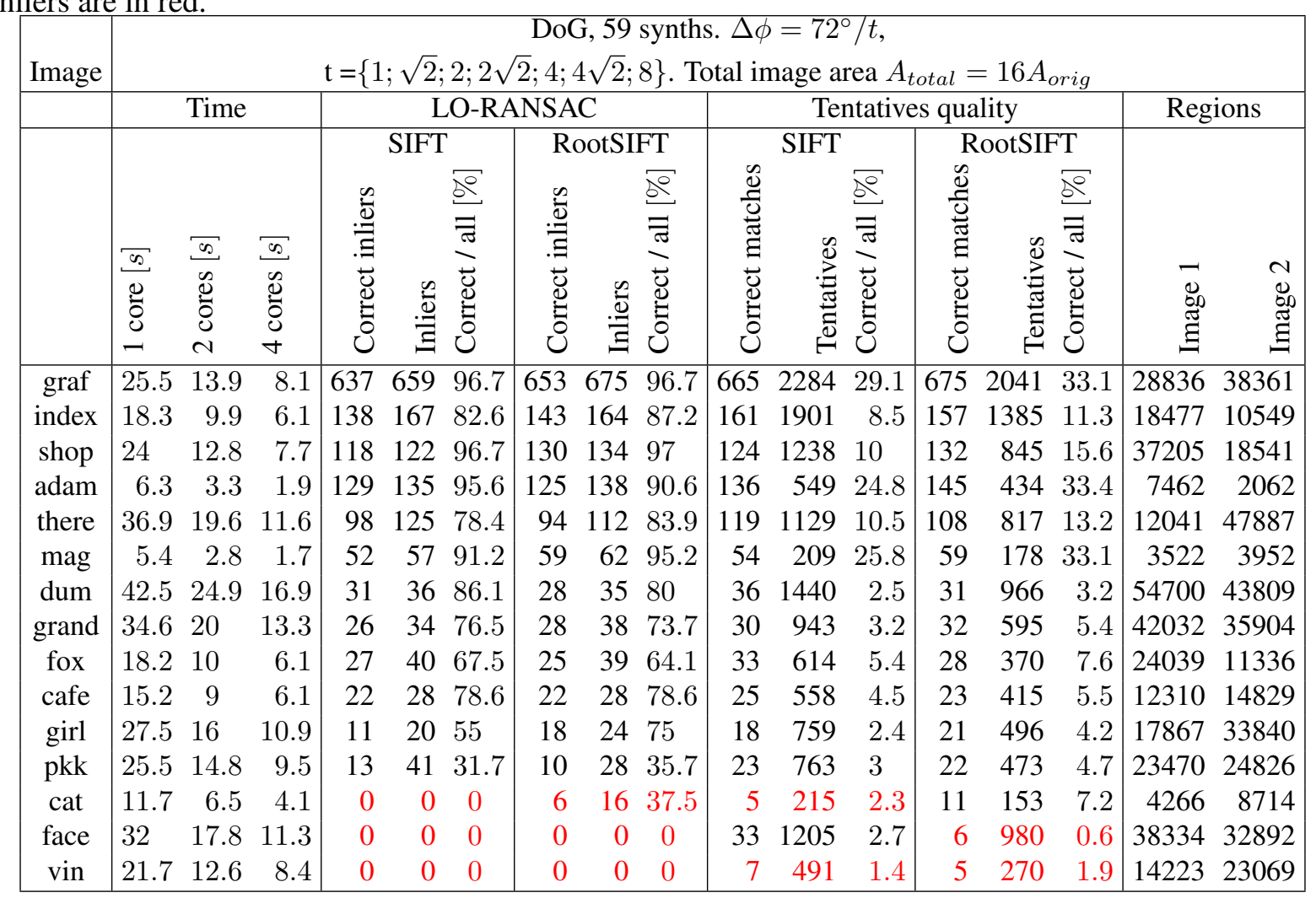


Table 17. Performance on the EVD dataset.ASIFT. Results with less than 8 correct inliers are in red.

\begin{tabular}{|c|c|c|c|c|c|c|c|c|c|c|c|}
\hline \multirow[t]{4}{*}{ Image } & \multicolumn{11}{|c|}{$\begin{array}{c}\text { ASIFT, } 59 \text { synths. } \Delta \phi=72^{\circ} / t \\
\mathrm{t}=\{1 ; \sqrt{2} ; 2 ; 2 \sqrt{2} ; 4 ; 4 \sqrt{2} ; 8\} . \text { Total image area } A_{\text {total }}=16 A_{\text {orig }}\end{array}$} \\
\hline & \multicolumn{3}{|c|}{ Time } & \multirow{2}{*}{\multicolumn{3}{|c|}{$\begin{array}{l}\text { ORSA } \\
\text { SIFT }\end{array}$}} & \multirow{2}{*}{\multicolumn{3}{|c|}{$\begin{array}{c}\text { Tentatives quality } \\
\text { SIFT }\end{array}$}} & \multicolumn{2}{|c|}{ Regions } \\
\hline & & & & & & & & & & & \\
\hline & $\begin{array}{l}\frac{\omega}{0} \\
0 \\
0 \\
0\end{array}$ & $\begin{array}{l}\sigma \\
\tilde{\sigma} \\
\tilde{\omega} \\
\tilde{0} \\
0 \\
\sim\end{array}$ & 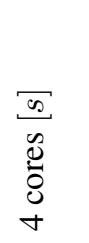 & 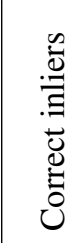 & $\stackrel{\mathscr{\Xi}}{\Xi}$ & 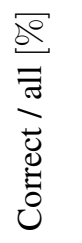 & 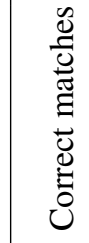 & 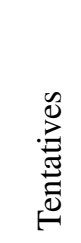 & 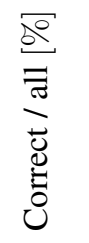 & $\begin{array}{l}\vec{D} \\
\infty \\
\tilde{\Xi} \\
\vec{\Xi}\end{array}$ & \\
\hline graf & 81.8 & 26.5 & 14.8 & 322 & 531 & 60.6 & 325 & 582 & 55.8 & 31199 & 38677 \\
\hline dex & 54.1 & 18.3 & 10.9 & 23 & 94 & 24.5 & 23 & 178 & 12.9 & 349 & 10115 \\
\hline & 79.5 & 25 & 14.1 & 17 & 34 & 50 & 18 & 76 & 23.6 & 41984 & 25270 \\
\hline $\mathrm{m}$ & 17.8 & 6 & 4.3 & 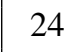 & 63 & 38.1 & 25 & 92 & 27.1 & 7572 & 3295 \\
\hline here & 150 & 48.4 & 27.8 & 20 & 72 & 27.8 & 21 & 365 & 5.8 & 26901 & 52334 \\
\hline $\mathrm{mag}$ & 16.1 & 5.5 & 3.8 & 11 & 25 & 44 & 12 & 54 & 22.2 & 4204 & 6399 \\
\hline dum & 158 & 50.8 & 48.3 & 3 & 39 & 7.7 & 3 & 64 & 4.7 & 66380 & 48622 \\
\hline grand & 131 & 41.8 & 40.4 & 0 & 0 & 0 & 1 & 81 & 1.2 & 54350 & 43713 \\
\hline fox & 47.4 & 15.7 & 9.5 & 0 & 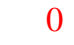 & 0 & 4 & 32 & 12.5 & 22300 & 13502 \\
\hline caf & 39.2 & 12.9 & 8 & 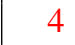 & 74 & 5. & 4 & 10 & 3.6 & 16088 & 16245 \\
\hline 511 & 110 & 35.6 & $20 . £$ & 0 & 0 & 0 & 12 & 19 & 6 & 35834 & 46892 \\
\hline pk & & 25.1 & 14.9 & 0 & U & U & 6 & 107 & 5.6 & 33229 & 22352 \\
\hline 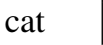 & 36.2 & 12.6 & 7.8 & 3 & 37 & 8.1 & 0 & 42 & 14.3 & 4979 & 10142 \\
\hline fac & 138 & 44.1 & 25.4 & 0 & 0 & 0 & 6 & 136 & 4.4 & 59278 & 41859 \\
\hline vin & 66.9 & 21.3 & 20.6 & 0 & 0 & 0 & 0 & 49 & 0 & 17127 & 31329 \\
\hline
\end{tabular}

Table 18. Performance on the EVD dataset. MODS $\left(\theta_{m}=15\right)$, SIFT. Results with less than 8 correct inliers are in red.

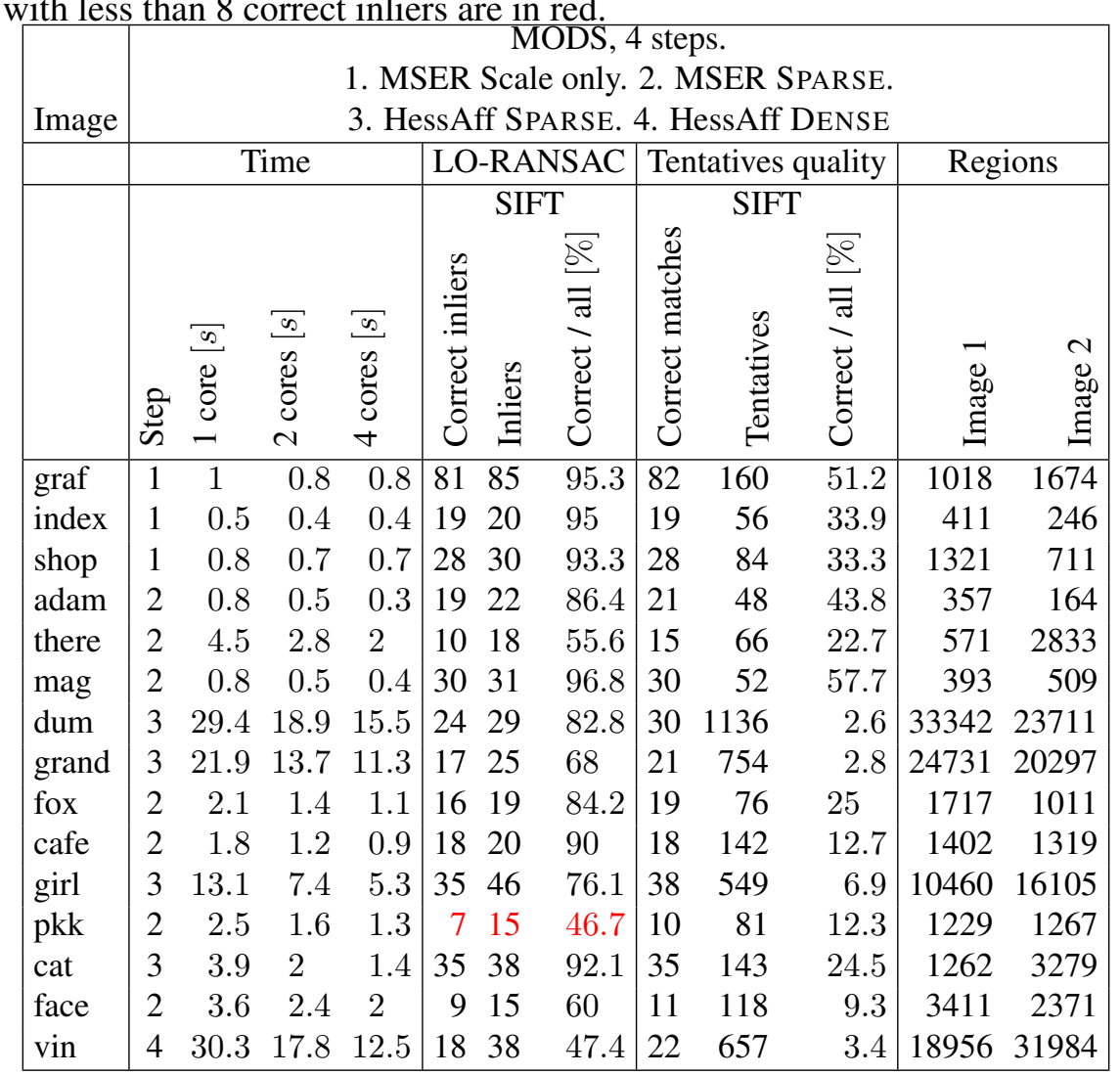


Table 19. Performance on the EVD dataset.MODS $\left(\theta_{m}=15\right)$, RootSIFT. Results with less than 8 correct inliers are in red.

\begin{tabular}{|c|c|c|c|c|c|c|c|c|c|c|c|c|}
\hline \multirow[t]{4}{*}{ Image } & \multicolumn{12}{|c|}{$\begin{array}{l}\text { MODS, } 4 \text { steps. } \\
\text { 1. MSER Scale only. 2. MSER SPARSE. } \\
\text { 3. HessAff SPARSE. 4. HessAff DENSE }\end{array}$} \\
\hline & \multicolumn{4}{|c|}{ Time } & \multirow{2}{*}{\multicolumn{3}{|c|}{\begin{tabular}{|c} 
LO-RANSAC \\
RootSIFT
\end{tabular}}} & \multicolumn{3}{|c|}{ Tentatives quality } & \multicolumn{2}{|c|}{ Regions } \\
\hline & & & & & & & & & $\operatorname{Root}^{5}$ & & & \\
\hline & 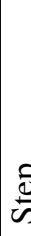 & $\begin{array}{l}\frac{\omega}{\infty} \\
\tilde{0} \\
0 \\
-1\end{array}$ & 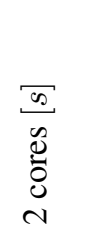 & $\begin{array}{l}\tilde{\sigma} \\
\tilde{u} \\
\tilde{0} \\
\dot{\sigma} \\
\sigma\end{array}$ & 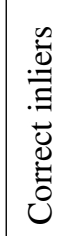 & $\stackrel{\mathscr{\Xi}}{\Xi}$ & 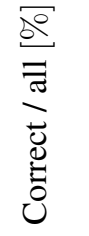 & 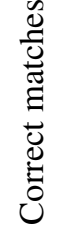 & 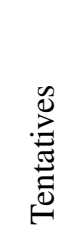 & 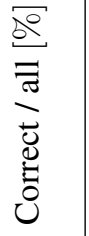 & 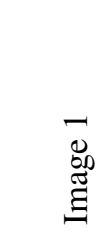 & 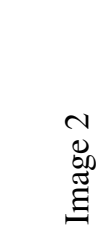 \\
\hline graf & 1 & & & 0.8 & 82 & 87 & 94.3 & 83 & 154 & 53.9 & 1018 & 1674 \\
\hline index & 1 & & 0.4 & 0.4 & 18 & 20 & 90 & 18 & 42 & 42.9 & 411 & 246 \\
\hline shop & 1 & 0.8 & 0.7 & 0.7 & 29 & 31 & 93.5 & 29 & 61 & 47.5 & 1321 & 71 \\
\hline adam & 2 & 0.8 & 0.5 & 0.4 & 20 & 23 & 87 & 22 & 47 & 46.8 & 357 & 164 \\
\hline there & 2 & 4.5 & 2.8 & 2 & 14 & 17 & 82.4 & 16 & 60 & 26.7 & 571 & 2833 \\
\hline mag & 2 & 0.9 & 0.5 & 0.4 & 31 & 31 & 100 & 31 & 44 & 70.5 & 393 & 509 \\
\hline dum & 3 & 27.2 & 16.9 & 13.5 & 25 & 32 & 78.1 & 29 & 850 & 3.4 & 33342 & 23711 \\
\hline grand & 3 & 20.9 & 12.5 & 10 & 14 & 24 & 58.3 & 19 & 468 & 4.1 & 24731 & 20297 \\
\hline fox & 2 & 2.1 & 1.4 & 1.1 & 19 & 20 & 95 & 20 & 62 & 32.3 & 1717 & 101 \\
\hline cafe & 2 & 1.8 & 1.2 & 0.9 & 17 & 21 & 81 & 18 & 117 & 15.4 & 1402 & 1319 \\
\hline girl & 3 & 13.1 & 7.3 & 5.2 & 34 & 44 & 77.3 & 38 & 436 & 8.7 & 10460 & 16105 \\
\hline pkk & 3 & 9.5 & 5.3 & 4 & 27 & 37 & 73 & 33 & 344 & 9.6 & 10686 & 7085 \\
\hline cat & 3 & 3.6 & 2.1 & 1.5 & 25 & 34 & 73.5 & 30 & 149 & 20.1 & 1262 & 3279 \\
\hline face & 3 & 15.6 & 8.9 & 7.1 & 39 & 44 & 88.6 & 42 & 534 & 7.9 & 18857 & 13271 \\
\hline vin & 4 & 29.7 & 17.1 & 11.8 & 19 & 32 & 59.4 & 21 & 455 & 4.6 & 18956 & 31984 \\
\hline
\end{tabular}

